Published in final edited form as:

Photochem Photobiol. 2005 ; 81(2): 215-237.

\title{
Molecular Contrast Optical Coherence Tomography: A Reviewף
}

\author{
Changhuei Yang* \\ Electrical Engineering Department, Engineering and Applied Sciences Division, California Institute of \\ Technology, Pasadena, CA
}

\begin{abstract}
This article reviews the current state of research on the use of molecular contrast agents in optical coherence tomography (OCT) imaging techniques. After a brief discussion of the basic principle of OCT and the importance of incorporating molecular contrast agent usage into this imaging modality, we shall present an overview of the different molecular contrast OCT (MCOCT) methods that have been developed thus far. We will then discuss several important practical issues that define the possible range of contrast agent choice, the design criteria for engineered molecular contrast agent and the implementability of a given MCOCT method for clinical or biological applications. We will conclude by outlining a few areas of pursuit that deserve a greater degree of research and development.
\end{abstract}

\section{Abbreviations}

ANSI, American National Standard; bR, bacteriorhodopsin; CARS, coherent Anti-Stokes Raman Scattering; FDA, Food and Drug Administration; FDOCT, Fourier domain OCT; GFP, green fluorescent protein; ICG, indocyanine green; MCOCT, molecular contrast optical coherence tomography; NIVI, nonlinear interferometric vibrational imaging; OCT, optical coherence tomography; phyA, phytochrome A; PPOCT, pump-probe OCT; SHG, second harmonic generation; SH-OCT, second harmonic OCT; SNR, signal-to-noise ratio

\section{INTRODUCTION}

Imaging methods that have the ability to detect specific molecular contrast agents, such as positron emission tomography (1), metallic ion-based magnetic resonance imaging (2) and fluorescence contrast microscopy, are vital imaging tools for a wide spectrum of biological research. The capability to map out contrast agent distribution within a biological sample provides a very important dimension of information beyond the tissue morphology-a biochemical map of the sample in question.

Of these imaging techniques, fluorescence contrast microscopy (3-7) stands out in terms of its ease of use, low cost and, most importantly, its wide applicability. For example, techniques based on Forster resonance energy transfer $(8,9)$ can be used to study the interaction dynamics of molecules within a cell. Fluorescence correlation spectroscopy is an excellent approach for studying the energy states and their associated transition lifetimes of biochemicals $(10,11)$. Alternatively, it can be used to study the diffusion dynamics of molecules in a medium. Evanescent wave fluorescence microscopy technique $(12,13)$, which excites fluorophore in a very narrow $(\sim 100 \mathrm{~nm})$ two-dimensional plane, is very useful for studying biochemical

\footnotetext{
TPosted on the website on 9 December 2004

*To whom correspondence should be addressed: Electrical Engineering Department, Engineering and Applied Sciences Division, California Institute of Technology, 1200 East California Boulevard, MC: 136-93, Pasadena, CA 91125, USA. Fax: 626-395-2137; email: chyang@caltech.edu.
} 
binding, chemical or object transfer dynamics on cell membranes. With the invention of antibody-conjugated fluorescence dye molecules (14), biologists have been able to tag specific protein type and study the protein function, protein transport and gene expression and regulation within a cell or an organ. The development of methods for expressing fluorescent protein, such as green fluorescent protein (GFP) (15-18) and Discosoma species red (19), endogenously by genetic manipulation solidly cements the role of fluorescence contrast microscopy in biological research. The applications of fluorescent contrast agents extend beyond basic research in biomedicine. Food and Drug Administration (FDA)-approved fluorescent dyes, such as indocyanine green (ICG) $(20,21)$ and fluorescein (22), have all found important applications in the clinical environment.

Fluorescence is not the only light-matter interaction mechanism that can be exploited for extracting biochemical information. The range of possible light-matter interaction mechanism, that includes spectrally dependent absorption, Raman scattering $(23,24)$ and second (and higher) harmonic generation (SHG) (25-27), can all be used to obtain specific biochemical information in a biomedical context. These optical phenomena form the basis for near-IR pulse oximeters, SHG microscopes $(25,27)$ and coherent Anti-Stokes Raman Scattering (CARS) microscopes (28-30). The richness of the possible light-matter interaction mechanisms and the low relative cost of the optical sources and detection systems imply that optically based techniques are well suited for performing molecular contrast-based imaging in a biomedical context.

One major disadvantage associated with optical bioimaging techniques is that they tend to have fairly shallow imaging penetration depth. This is attributed to the fact that biological tissues are highly scattering in the optical regime. For example, in the case of fluorescence microscopy, the emitted fluorescence photons from a specific location within tissue will undergo a significant amount of scattering events that randomize their direction of propagation as they make their way to the tissue surface. This makes it difficult to distinguish the fluorescence contribution from a specific volume within the tissue from the contribution from the neighboring volumes and thus degrades the image quality.

Two-photon fluorescence microscopy techniques (3), which enhance fluorescence within a specific scan volume, can do a much better job of profiling the fluorophore distribution within a tissue sample. However, the depth penetration of two-photon fluorescence techniques is still fairly limited. The penetration depth varies based on the turbidity (scattering property) of the targeted tissue; a typical maximum depth penetration of about 300-400 $\mu \mathrm{m}$ can be expected for images that still preserves an axial resolution that is largely defined by the confocal parameter.

In this respect, optical coherence tomography (OCT) (31-36) compares very favorably with fluorescence-based or any other optically based imaging techniques; imaging depth of millimeters with spatial resolution of a couple of microns are relatively easily achieved with OCT at light fluence levels that do not exceed American National Standard (ANSI)recommended limits (37). OCT's superior depth penetration and high axial spatial resolution is attributable to its ability to selectively detect only the ballistically propagating components (38) of the backscattered light originating from a specific selectable depth within the sample. These components are the fraction of the light that are not scattered or deflected to any extent by the tissue.

OCT can be thought of as the optical equivalent of ultrasound tomography; both techniques generate reflectivity-based images of the target sample by measuring the returning echo response to a specific input pulse or spectral signal. The ability of OCT to render high-quality three-dimensional structural images of biological targets noninvasively has propelled its rapid 
adaptation for clinical applications, most notably in imaging the anterior and posterior segments of the eye (39-43), vascular tissues $(44,45)$ and gastrointestinal tracts $(46-48)$.

A hybrid optical imaging approach that can combine OCT's ability to perform high-resolution and excellent penetration depth imaging with fluorescence contrast microscopy's ability to elicit molecular contrast from the sample can dramatically enhance the capability of clinicians and biomedical researchers to track biochemical distribution and changes within patients and experimental subjects.

However, the recognition of the fact that a hybrid approach can dramatically change the landscape of biomedical imaging has recently prompted several research groups to implement various modified OCT schemes that have the capability to detect molecular contrast agents or contrast agents that can potentially bind to a specific chemical or protein.

There are two ways in which molecular contrast-based OCT (MCOCT) techniques can potentially be used to image a specific chemical or protein distribution within a target. The first approach is to simply have the MCOCT method directly detect the chemical or protein. This does require the chemical or protein to perform well as a contrast agent. The second approach for contrast imaging is more generally applicable; a contrast agent that is easily detected by the MCOCT method can be functionalized so that it binds to the specific chemical or protein of interest. In the case where the target of interest is a protein type, such functionalization can be accomplished by conjugating the contrast agent to an appropriate antibody (49). This approach has a very significant advantage in that chemical or protein that is otherwise undetectable directly by optical means can still be detected and mapped in the target, as long as it is possible to design a contrast agent that can bind to it.

The scope of this review will be confined to the discussion of MCOCT methods and the contrast agents that the methods are designed to detect. MCOCT research is at an early stage of development; consequently, there are no reported researches into the functionalizing of MCOCT contrast agents yet.

We begin the review with a brief introduction to some key concepts in OCT. We will then discuss the three major classes of approaches that researchers have taken to implement MCOCT techniques. Next, we will summarize the various reported techniques that have been implemented thus far. We will then discuss several important considerations that define the possible range of contrast agent choice, the design criteria for MCOCT methods and the implementability of a given MCOCT method for clinical or biological applications. Finally, we will conclude by outlining a few areas of pursuit that deserve a greater degree of research and development.

\section{BACKGROUND}

A basic OCT (31-36) scheme is shown in Fig. 1. In such a scheme, a broadband light source is coupled into an interferometer and the light is split into two components at the coupler. One component (reference beam) is reflected from a reference mirror, whereas the other (probe beam) is focused on the target sample. The backscattered light from various depths within the sample is collected and recombined with the reference reflection, and the resulting interference signal is monitored with a photodetector.

The amount of spatially coherent light that is backscattered and collected from a given depth in the sample, $z_{s}$, can be expressed as: 


$$
P_{S}\left(\lambda, z_{s}\right)=P_{\mathrm{So}} \mathrm{e}^{-2 \int_{0}^{z} s_{0}\left[\mu_{d}\left(\lambda, z_{s}^{\prime}\right)+\mu_{s}\left(\lambda, z_{s}^{\prime}\right)\right] d z_{s}^{\prime}} R\left(\lambda, z_{s}\right)
$$

where $P_{\text {So }}$ is the input probe beam power, $\mu_{a}\left(\lambda, z_{s}^{\prime}\right)$ is the absorption extinction coefficient of the sample at depth $z_{s}^{\prime}, \mu_{s}\left(\lambda, z_{s}^{\prime}\right)$ is the scattering extinction coefficient of the sample at depth $z_{s}^{\prime}$ and $R\left(\lambda, z_{s}\right)$ is the fractional reflectivity of the sample at depth $z_{s}$ and wavelength $\lambda$. In general, there is an additional multiplicative factor that accounts for the light collection geometry of the imaging system. This factor is strongly dependent on the optical system design involved and is not relevant to our discussion of MCOCT methods. For simplicity, we shall assume the factor to be unity for the rest of this review.

The interference signal, $P_{\text {interference }}(\lambda, z)$, at a center wavelength, $\lambda$, as a function of the scanning reference arm's displacement $z$, can be expressed as:

$$
\begin{aligned}
& P_{\text {interference }}(\lambda, z)=2 \sqrt{P_{\mathrm{Ro}}} \int_{0}^{\infty} \sqrt{P_{S}\left(\lambda, z_{s}\right)} \cos \left(\frac{4 \pi}{\lambda}\left(z-z_{s}\right)\right) \\
& \mathrm{e}^{-\frac{[2 \ln (2)]^{2}\left(z-z_{s}\right)^{2}}{l_{c}^{2}} d z_{s}} \\
& =2 \sqrt{P_{\mathrm{Ro}}} \sqrt{P_{s}(\lambda, z)} \otimes \cos \left(\frac{4 \pi}{\lambda} z\right) \mathrm{e}^{-\frac{[2 \ln (2)]^{2} z^{2}}{I_{c}^{2}}} \\
& =2 \sqrt{P_{\mathrm{Ro}} P_{\mathrm{So}}} \mid \mathrm{e}^{-\int_{0}^{z_{0}\left[\mu_{a}\left(\lambda, z_{s}{ }^{\prime}\right)+\mu_{s}\left(\lambda, z_{s}{ }^{\prime}\right)\right] d z_{s}{ }^{\prime}} \sqrt{R(\lambda, z)}} \\
& \otimes \cos \left(\frac{4 \pi}{\lambda} z\right) \mathrm{e}^{-\frac{[2 \ln (2)]^{2} z^{2}}{I_{c}^{2}}}
\end{aligned}
$$

where $P_{\text {Ro }}$ is the collected reference arm power, $P_{S}\left(\lambda, z_{S}\right)$ is the collected signal arm power from the sample at a depth of $z_{s}, P_{\mathrm{So}}$ is the collected signal arm power if the sample is fully reflective, $R\left(\lambda, z_{s}\right)$ is the sample reflectivity at depth $z_{s}$, the additional exponential term accounts for the additional loss of collected light because of absorption (modeled by the extinction coefficient $\mu_{\mathrm{a}}\left(\lambda, \mathrm{z}_{\mathrm{s}}\right)$ and scattering (modeled by the scattering extinction coefficient $\mu_{\mathrm{s}}\left(\lambda, \mathrm{z}_{\mathrm{s}}\right)$ during the passage into and out of the sample. $l_{\mathrm{c}}$ is the source coherence length and is a function of the source's bandwidth: $l_{c}=\ln (2)\left((2 / \pi)\left(\lambda^{2} / \Delta \lambda\right)\right)(50)$, where $\lambda$ is the center wavelength and $\Delta \lambda$ is the bandwidth of the light source. The second part of Eq. (2) shows that the detected signal can be written as the convolution of the sample's reflectivity profile with the coherence envelop as expressed by the last term of the convolution.

By translating the reference mirror at a uniform speed and filtering the acquired signal at the induced Doppler shift frequency associated with the translation of the reference mirror, we can generate a depth-resolved profile (termed an A-scan) of the sample's reflectivity with an axial resolution equal to the source's coherence length:

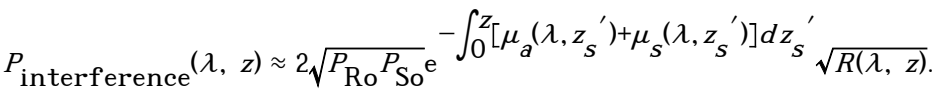

The dropping of the convolution kernel, $\cos ((4 \pi / \lambda) z) \mathrm{e}^{\left([2 \ln (2)]^{2} z^{2}\right) / l_{c}{ }^{2}}$, from Eq. (2) to get Eq. (3) is valid only if we restrict ourselves to axial length considerations that is no finer than the coherence length. 
By laterally translating the probe beam and sequentially acquiring a series of A-scans, a twodimensional depth-resolved image (B-scan) of the sample can be generated. The axial resolution of an OCT imaging system is defined by the source coherence length, and the lateral resolution is defined by probe beam size diameter. Interested readers are encouraged to refer to Ref. (35) for a more detailed explanation of OCT's operating principle.

At this point, we would like to highlight the distinction between the scattering extinction coefficient, $\mu_{s}\left(\lambda, z_{s}^{\prime}\right)$, and the reduced scattering extinction coefficient, $\mu_{s}^{\prime}\left(\lambda, z_{s}^{\prime}\right)(51,52)$ (see Fig. 2). For simplicity, consider a purely scattering and nonabsorptive medium. The scattering extinction coefficient characterizes the fraction of a plane wave that remains after passage through a scattering medium, whereas the reduced scattering extinction coefficient characterizes the fraction of transmitted optical power through a scattering medium. An oversimplified but intuitive way to draw the distinction is to equate the fractional remain of a propagating plane wave through a scattering medium to the fraction of ballistically propagating photons that did not experience any scattering during the passage, and to equate the fractional remain of the optical power transmission through the same scattering medium as the fraction of photons that make their way through the scattering medium. The second fractional remain is inclusive of the first and will also include photons that have been deviated in their propagation direction to some extent but are still traveling in a more or less forward direction. The distinction is important because light that has been scattered during the transmission process loses its spatial coherence (the light field wavefront is no longer planar) and will not interfere with the reference beam, even if it is collected by the detector. As such, the relevant scattering extinction coefficient for use with OCT calculation is $\mu_{s}\left(\lambda, z_{s}^{\prime}\right)$. The reduced scattering extinction coefficient is used to calculate the total power transmission to a specific depth in the sample. For situations where the spatial coherence is irrelevant, such as experimental conditions where we are simply using a light field to excite a dye, $\mu_{s}^{\prime}\left(\lambda, z_{s}^{\prime}\right)$ is the appropriate scattering extinction coefficient for use. Generally, light scattering within biological samples is highly forward directed, as such $\mu_{s}^{\prime}\left(\lambda, z_{s}^{\prime}\right)$ is typically much smaller than $\mu_{s}\left(\lambda, z_{s}^{\prime}\right)$ (typically $10 \times$ smaller) (51, 52). The above statements may be generalized for a scattering and absorptive medium by replacing the scattering coefficient with the attenuation coefficient, given by the sum of $\mu_{s}(\lambda, z$ $\left.{ }_{s}\right)$ and $\mu_{a}\left(\lambda, z_{s}^{\prime}\right)$, and by replacing the reduced scattering coefficient with the effective attenuation coefficient, given by the sum of $\mu_{s}^{\prime}\left(\lambda, z_{s}^{\prime}\right)$ and $\mu_{a}\left(\lambda, z_{s}^{\prime}\right)$.

Interferometric methods, such as OCT, can achieve shot noise limited detection sensitivity (50). This implies that the sensitivity of the method to detect a weak $P_{S}$ approaches the fundamental limit as set by the ideal situation where we directly measure $P_{S}$ with a perfectly noiseless detector. The interferometric signal-to-noise ratio (SNR) can be expressed as:

$$
\begin{aligned}
& \mathrm{SNR}=\left[\left(\varepsilon \frac{1}{2} P_{\text {interference }}(\lambda, z) T / \mathrm{h} v\right) / \sqrt{\left(\varepsilon P_{\mathrm{Ro}} T / \mathrm{h} v\right)}\right]^{2} \\
& =\frac{\varepsilon P_{S}(\lambda, z) T}{\mathrm{~h} v},
\end{aligned}
$$

where $\varepsilon$ is the detection efficiency and $h v$ is the energy quantum of the OCT light source. Equation 4 is derived by squaring the ratio of the number of interference signal photons detected over a given measurement time $T$ (numerator of the first line of the equation) to the number of shot noise photons detected within the same time frame (denominator of the first line of the equation). The measurement time is equal to the length of the timebase that is invested in the acquisition of a single spatial image pixel. The shot noise strength is simply proportional to the standard deviation of the total signal present, which in this case is dominated by the reference arm power $P_{\text {Ro }}$. This SNR value is the same magnitude as that associated with the direct detection of $P_{S}$ with a noiseless detector: 


$$
\begin{aligned}
& \mathrm{SNR}_{\text {direct }}=\left[\left(\varepsilon P_{s}(\lambda, z) T / \mathrm{h} v\right) / \sqrt{\left(\varepsilon P_{s}(\lambda, z) T / \mathrm{h} v\right)}\right]^{2} \\
& =\frac{\varepsilon P_{s}(\lambda, z) T}{\mathrm{~h} v} .
\end{aligned}
$$

The shot noise term is larger in the interferometric method, but that is compensated by the amplification of the detected signal because of interference.

Making light field measurements using interferometry rather than direct detection has its advantages. First, interferometry methods allow for shot noise limited detection even when the detectors used have high dark current noise; $P_{\text {Ro }}$ simply needs to be sufficiently large, such that the shot noise term dominates over the dark current term. The tolerance to noisy detectors is especially relevant in IR or near-IR regime imaging applications where low-noise photomultiplier tubes are simply not available. Second, interferometry methods are extremely well suited to the task of detecting a specific light component out of many. If a given light component is not coherent with the reference component, it simply will not interfere and contribute a detectable signal. This implies that stray light is almost always rejected in interferometry detection; in the case of OCT, the broad bandwidth of the input light source implies that light components from the original light source will also be rejected if they did not travel the same optical distance as the reference component.

The shot noise limited detection achieved by OCT implies that it is well suited for detecting the weak backscattered light from tissues. A typical OCT system can achieve an SNR of more than $100 \mathrm{~dB}$, which implies that the system is sensitive enough to detect a single backscattered photon from an incident input light field to the sample that consists of $10^{10}$ photons. In addition to the sensitivity, OCT's superior axial gating can efficiently eliminate noisy contribution by backscattered light from above or below the targeted gated region. The combination of these two advantages enables OCT to achieve an imaging depth of millimeters in biological targets.

Recently, a different OCT imaging approach, Fourier domain OCT (FDOCT), has been developed and demonstrated to be even more sensitive than the traditional time domain OCT methods (such as the one depicted above in Fig. 1). FDOCT (53) has also been termed spectral radar (54) and spectral-domain OCT $(21,55)$. FDOCT differs from time domain OCT in that the detected light is spectrally resolved. One form of FDOCT spectrally disperses the light within a spectrometer and detects the spectrally resolved components using $\mathrm{N}$ different detectors or channels. N can range from 100 to 1000 depending on the spectrometer used. Unlike in time domain OCT, the reference arm length is kept unchanged during the image acquisition process. The Fourier transform of the measured spectral profile is a depth-resolved profile of the sample. This A-scan is similar to that measured by time domain OCT, except that the SNR is improved by a factor equal to N/2 (56-58). Another form of FDOCT uses a monochromatic laser source that is swept in its wavelength during the signal acquisition process $(21,56,59)$; the method leads to an equivalent SNR improvement. The improvement in the SNR for FDOCT is attributable to the much longer time window during which the signal associated with a single spatial pixel is acquired. In the case of time domain OCT, this time window is equal to the short time duration when the reference arm is transiently matched to the particular sample depth of interest during the scan. In comparison, this time window is equal to the entire duration of the scan acquisition for FDOCT.

Thus far, the reported implementations of MCOCT methods have all been based on time domain OCT systems. However, the adaptations of the MCOCT methods, which have been reported so far, into FDOCT formats are fairly straightforward. All the methods can benefit dramatically in terms of contrast agent sensitivity through such an adaptation. Although the concepts in this article do not require a detailed understanding of FDOCT, interested readers are encouraged to read Refs. (56-58) to better understand FDOCT. 
The rapid pace of research development in OCT technology has consistently improved on the quality, speed and sensitivity of OCT imaging systems. At present, the highest resolution reported is $0.5 \mu \mathrm{m}$, and it was achieved with the use of a $325 \mathrm{~nm}$ wide optical spectrum at 725 $\mathrm{nm}(60)$. The fastest reported scan rate to date is 31 frames per second for a frame size of 1024 $\times 512$ pixels $(61)$.

It is clear from the expression for the OCT signal (Eq. 2) that OCT primarily measures the reflectivity profile of the sample, and therefore, its image information is structural in nature. Methods for eliciting molecular contrast distribution information with an OCT imaging system are constrained by the detection boundary of interferometry. As an example, let us consider fluorescence. Fluorescence is an incoherent optical process, which implies that it has no optical phase relationship with its excitation light field, and as such it cannot be detected in any realizable OCT detection scheme. Therefore, it is impossible to design an MCOCT method that is capable of detecting fluorescence signal directly.

MCOCT schemes, that have been reported to date, can be broadly categorized into three major groups. The first group uses the absorption properties of the contrast agents to elicit contrast. This group can be further divided into two subcategories. The first subcategory maps out the contrast agent distribution by changing the absorption spectrum of a specific molecular contrast agent that has been introduced into the biological target and acquiring a pair of OCT scans before and after the change. The difference of the two OCT scans can then be processed to reveal the distribution of the contrast agent. The second subcategory passively interrogates and maps out the contrast agent's distribution by making use of the contrast agent's absorption spectral profile. The second major group of MCOCT schemes is based on the use of molecular contrast agent, which can efficiently and coherently convert an illumination light field into an emission light field that is amendable to interferometry detection. The third group makes use of the unique scattering properties of specially designed contrast agent to profile the contrast agent's distribution in the target. The next three sections will look at examples of each group in greater detail.

\section{ABSORPTION-BASED MCOCT METHODS}

There are four reported MCOCT methods that fall within this category: pump-probe MCOCT (62), pump-suppression MCOCT (63), spectroscopic OCT $(64,65)$ and spectral triangulation MCOCT (66). All these techniques rely on introducing a molecular contrast agent with a specific optically excitable transition or a well-identified absorption spectrum into the target sample.

\section{Optically excitable transition MCOCT methods}

The general concept for extracting the contrast agent distribution within the sample with the first two methods is straightforward (see Fig. 3). In both cases, a baseline OCT scan of the sample containing the contrast agent is first acquired. Next, the contrast agent is optically altered so that its absorption spectrum is changed. A second OCT scan is then acquired. The two OCT scans will appear slightly different; specifically, the extinction coefficient $\mu_{a}(\lambda, z)$ will be different because the contrast agent contributes significantly to it. Mathematically, the extinction coefficient, in this context, can be written as:

$$
\begin{aligned}
& \mu_{a}(\lambda, z)=\mu_{a, \text { intrinsic }}(\lambda, z)+\mu_{a, \text { contrast_agent_baseline }}(\lambda, z) \\
& +\Delta \mu_{a, \text { contrast_agent }}(\lambda, z),
\end{aligned}
$$

where $\mu_{a \text {,intrinsic }}(\lambda, z)$ is the intrinsic absorption extinction coefficient of the sample at location $z$ and wavelength $\lambda$, $\mu_{\mathrm{a} \text {,contrast_agent_baseline }}(\lambda, \mathrm{z})$ is the localized corresponding absorption 
extinction coefficient of the sample that is attributable to the contrast agent for the first OCT scan (baseline), and $\Delta \mu_{a \text {,contrast_agent }}(\lambda, z)$ is the corresponding change in the absorption extinction coefficient of the sample between the two OCT scans. The last two terms can, in turn, be reexpressed as a function of the baseline absorption cross-section of the molecule $\left(\sigma_{\text {baseline }}(\lambda, z)\right)$, the change in absorption cross-section on alteration $(\Delta \sigma(\lambda, z))$, the localized number concentration of the molecules $(n(z))$ and the localized number concentration of the molecules altered by the optical excitation $(\Delta n(z))$ :

$$
\begin{gathered}
\mu_{a, \text { contrast_agent_baseline }}(\lambda, z)=n(z) \sigma_{\text {baseline }}(\lambda, z) \\
\Delta \mu_{a, \text { contrast_agent }}(\lambda, z)=\Delta n(z) \Delta \sigma(\lambda)
\end{gathered}
$$

The procedure for extracting the contrast agent distribution in the sample simply involves calculating $\Delta n(z)$ from the two OCT scans. Note that the actual concentration of the contrast agent cannot be found; instead, the measurable quantity is the number concentration of molecules that are optically altered. An intermediary quantity $U(z)$, which is the number concentration integral to the depth of $z$ can be found with minimal processing of the two scans:

$$
\begin{aligned}
& U(z)=\frac{1}{\Delta \sigma(\lambda)} \ln \left(\frac{P_{\text {interference, baseline }}(\lambda, z)}{P_{\text {interference, excited }}(\lambda, z)}\right) \\
& =\frac{1}{\Delta \sigma(\lambda)} \ln \left(\mathrm{e}^{-\int_{0}^{z}\left[\mu_{a, \text { contrast_agent_baseline }}\left(\lambda, z_{s}{ }^{\prime}\right)+\mu_{s}\left(\lambda, z_{s}{ }^{\prime}\right)\right] d z_{s}{ }^{\prime}}\right. \\
& \times \mathrm{e}^{\left.-\int_{0}^{z}\left[\mu_{a, \text { contrast_agent_baseline }}\left(\lambda, z_{s}{ }^{\prime}\right)+\Delta \mu_{a, \text { contrast_agent }}\left(\lambda, z_{s}{ }^{\prime}\right)+\mu_{s}\left(\lambda, z_{s}{ }^{\prime}\right)\right] d z_{s}{ }^{\prime}\right)} \\
& =\frac{1}{\Delta \sigma(\lambda)} \int_{0}^{z} \Delta \mu_{a, \text { contrast_agent }}\left(\lambda, z_{s}{ }^{\prime}\right) d z_{s}{ }^{\prime} \\
& =\int_{0}^{z} \Delta n\left(z_{s}{ }^{\prime}\right) d z_{s}{ }^{\prime},
\end{aligned}
$$

where $P_{\text {interference,baseline }}(\lambda, z)$ is the baseline OCT scan signal and $P_{\text {interference,excited }}(\lambda, z)$ is the OCT scan signal after the contrast agent is optically altered. In this situation, $R(\lambda, z)$,

$\mu_{a, \text { contrast_agent_baseline }}\left(\lambda, z_{s}^{\prime}\right), \mu_{s}\left(\lambda, z_{s}^{\prime}\right)$ are assumed to be unchanged between the two scans. To obtain the localized distribution $\Delta n(z)$, we differentiate $U(z)$ with respect to $z$ :

$$
\Delta n(z)=\frac{d U(z)}{d z}
$$

There are several approaches for creating the necessary absorption cross-section change in the contrast agent by optical excitation. In the subsequent subsections, we shall see two examples of such approaches and one example in which the molecules are not optically excited but we can nevertheless elicit an absorption cross-section difference by modifying the imaging method.

Pump-probe OCT-Pump-probe OCT (PPOCT) (62) was the first reported approach for performing MCOCT imaging. The dye used in the initial demonstration was methylene blue, a dye that was used in stain bacteriology (67) and as a contrast agent in chromoendoscopy (68). The strategy used in PPOCT (see Fig. 4) is simple and direct, an optical excitation field changes the absorption cross-section of the dye at the OCT probe wavelength by shelving the molecules into a relatively long-lived triplet state.

More specifically, methylene blue (62), like numerous other dye species, has an associated set of triplet states that are relatively long lived (relaxation time of $\sim 2 \mu \mathrm{s}$ ). By using a pump light field at the molecules' singlet absorption wavelength ( $650 \mathrm{~nm}$ in the case of methylene blue), 
the molecule can be forced to cycle between its ground and higher singlet states. There is a probability that the molecule will transit into one of the triplet states when it relaxes from the singlet state. The triplet-triplet absorption occurs in the $830 \mathrm{~nm}$ region, a suitable wavelength regime for an OCT probe choice.

In the limit where the pump intensity is weak, the total number concentration of molecules that are shelved into the triplet state under a steady state excitation field can be found to be:

$$
\Delta n(z) \approx(1-q) n_{o}(z) \frac{I_{\text {pump }}(z)}{I_{\text {sat }}},
$$

where $n_{o}(z)$ is the total number concentration of the molecules at depth $z, q$ is the quantum efficiency of the fluorescence process, $I_{\text {pump }}(z)$ is the pump intensity at depth $z$ and $I_{\text {sat }}$ is the saturation intensity of the molecules. $I_{\text {sat }}$ is given by $I_{\text {sat }}=\mathrm{h} v_{g-s} / \sigma_{g-s} \tau_{\text {singlet }}$, where $\mathrm{h} v_{g-s}$ is the energy quanta of the pump light source, $\sigma_{g-s}$ is the absorption cross-section associated with the ground to singlet state transition and $\tau_{\text {singlet }}$ is the decay time constant for the singlet to ground state transition. The derivation of Eq. (10) can be found in Appendix 1.

The reported implementation of the method used a Q-switched Nd:YAG laser to provide the necessary excitation field. Its pulsed nature and high energy per pulse ensured that the instantaneous excitation field intensity was high. On the basis of the numbers provided in the report, the calculated maximum instantaneous intensity was $16 \mathrm{MW} / \mathrm{cm}^{2}$ at the sample's surface. This intensity can be expected to drop as a function of depth into the sample. In the limit where the drop off is insignificant, the MCOCT signal as calculated based on Eq. (10) will be a fairly good measure of the contrast agent's distribution profile within the sample because the fraction of shelved molecules will be a constant fraction of the total molecules. This is a fairly reasonable assumption because the transmission intensity drop off is proportional to the effective attenuation coefficient of the sample (typically 10 times smaller than the scattering extinction coefficient for biological samples). In the event that the drop off is deemed to be significant, the contrast map is nevertheless a good indication of the contrast agent distribution that can be rescaled appropriately if the reduced scattering extinction coefficient is known.

Figure 4 shows the MCOCT images acquired with this particular implementation approach. An OCT probe at center wavelength of $800 \mathrm{~nm}$ and bandwidth of $90 \mathrm{~nm}$ was used to acquire the OCT image before and after the pump field excitation. The contrast agent concentration used in the experiment was about $500 \mu M$, and the researchers were able to detect its presence to a depth of about $0.5-0.7 \mathrm{~mm}$ in a scattering medium consisting of $0.5 \%$ Liposyn solution.

The major challenge with this technique lies in creating a sufficiently fast OCT imaging system to acquire the OCT signal with the dye molecules in their shelved states. Although the triplet state lifetime of $2 \mu \mathrm{s}$ is long in the context of molecular state transitions, it is, nevertheless, a short time duration in the context of pump-probe-based imaging techniques. With such a short time duration, the researchers had only a sufficient time window to acquire a single OCT image pixel per pump-probe cycle. The efficiency of the system can presumably be dramatically improved by sustaining the excitation field during the entire later half of the acquisition cycle. However, that can be expected to lead to a high light fluence onto the sample. This matter is compounded by the fact that a small $\tau_{\text {singlet }}$ leads to a high $I_{\text {sat }}$ value, which implies that the pump intensity has to be fairly high to achieve a reasonably efficient shelving of molecules into the triplet state (see Eq. 10). 
Pump-suppression MCOCT-This next class of MCOCT seeks to address the above issues associated with the short transition duration of the molecular contrast agents by choosing a slightly different molecular transition mechanics-conformational change of molecules.

Two examples of such molecular candidates are bacteriorhodopsin (bR) (69) and phytochrome A (PhyA) (70-72). Both molecules undergo a conformational change on excitation by a light field of the appropriate wavelength. These two molecular candidates are very attractive MCOCT contrast agent candidates because they are proteins. In principle, it should be possible to genetically engineer animal models to express these proteins for biomedical research applications; they can fulfill the roles in MCOCT that the various fluorescent proteins are serving for fluorescence microscopy applications.

Of the two candidate protein types, phyA proved to be the more appropriate contrast agent for OCT application. It has a strong absorption peak in the near-IR regime for one of its molecular states, and the absorption peak shifts significantly when the molecules undergo a conformational change. The absorption peak is close to the operating wavelength range of OCT imaging systems. Reference (63) is a report on an MCOCT demonstration with phyA.

The basic mechanics of the molecules that is useful for this MCOCT method can be summarized as follows (see Fig. 5). Each molecule has two possible states (State A and B); State A is more stable than State B, and molecules in State B will revert into State A with a long time constant $\tau_{B \rightarrow A}$. State $A$ has an absorption maximum at a wavelength of $\lambda_{A}$, and State $B$ has the corresponding peak at a wavelength of $\lambda_{B}$. Upon excitation with light field at or near $\lambda_{A}$, the molecules will transit to state $\mathrm{B}$ and vice versa. In the case of phyA, the molecules have the following parameters: $\tau_{\mathrm{B} \rightarrow \mathrm{A}} \sim \mathrm{s}$, State $A=\operatorname{Pfr}$ state, State $B=\operatorname{Pr}$ state, $\lambda_{\mathrm{A}}=740 \mathrm{~nm}$, and State $B$ 's at a wavelength of $\lambda_{B}=670 \mathrm{~nm}$. The wavelength regime around the absorption maximum of Pfr state is appropriate for situating the probe OCT spectral band.

The strategy for MCOCT imaging in this situation is quite different from the case for PPOCT. First of all, the shifting of molecular population from one state to another no longer requires the use of a high-intensity light field. Because the relaxation time $\tau_{\mathrm{B} \rightarrow \mathrm{A}}$ is at least six orders of magnitude larger than $\tau_{\text {singlet }}$, the equivalent $I_{\text {sat }}$ is correspondingly lower by at least six orders of magnitude. The corresponding absorption state dye molecule concentration change when the molecules are illuminated with a light field at wavelength of $\lambda_{\mathrm{A}}$ can be expressed as:

$$
\begin{aligned}
& \Delta n(z)=\Delta n_{\operatorname{Pfr}}(z) \\
& =-n(z)\left(\frac{1}{2+\left(I_{\text {sat }} / I_{\text {pump }}(z)\right)}\right) \approx-\frac{n(z)}{2}
\end{aligned}
$$

where $n_{\mathrm{Pfr}}(z)$ is the number concentration of molecules in the Pfr state at a depth $z$ in the sample, $n(z)$ is the total number concentration of the contrast agent at depth $z$ and $I_{\text {pump }}(z)$ is the pump intensity at depth $z$. $I_{\text {sat }}$ is the saturation intensity and is given by $I_{\text {sat }}=h v / \tau_{\operatorname{Pr} \rightarrow \operatorname{Pfr}}$, where $h v$ is the energy quanta associated with the pump beam and $\sigma$ is the corresponding absorption crosssection. For this contrast agent, $I_{\text {sat }}$ is so low that a pump intensity of about $1 \mathrm{~W} / \mathrm{cm}^{2}$ is observed to be sufficient to drive the transition. The advantage of using low-intensity light to actuate state population changes is especially relevant in the context of clinical imaging where the ANSI guidelines (37) for the amount of laser light exposure have to be adhered to.

For the pump-suppression MCOCT demonstration with phyA, the imaging strategy used is as follows. A $750 \mathrm{~nm}$ OCT probe beam on the sample during the entire imaging process; in the absence of other illumination, phyA would be forced into its Pr state by the OCT probe beam (intensity of about $260 \mathrm{~W} / \mathrm{cm}^{2}$ ). The image acquisition process involved the following steps: (1) the $660 \mathrm{~nm}$ illumination that was about an order of magnitude more intense than the OCT 
probe beam was switched on; (2) after a pause of $500 \mathrm{~ms}$ to allow time for phyA to transit into its Pfr state, an averaged OCT scan was acquired; (3) the $660 \mathrm{~nm}$ light was turned off; and (4) after a pause of $500 \mathrm{~ms}$ to allow phyA to transit into its Pr state, an averaged Pr OCT scan was acquired. In effect, the $660 \mathrm{~nm}$ illumination in Step (A) served as a suppression pump that opposes the shifting of molecules into the Pr state by the OCT probe beam.

Figure 5 shows the MCOCT images acquired with this experimental scheme. The contrast agent concentration used in the experiment was $83 \mu M$, and the researchers were able to detect its presence to a depth of about $1.5 \mathrm{~mm}$ in a scattering medium consisting of $0.2 \%$ Intralipid solution. The experiment is notable for the low light intensities used. The OCT probe light intensity and the suppression pump intensity are $260 \mathrm{~W} / \mathrm{cm}^{2}$ and $1.1 \mathrm{~kW} / \mathrm{cm}^{2}$, respectively.

The advantage of this method in terms of the required probe and pump light intensity levels is significant. However, unlike in the case for the previous method, the choice of molecular contrast candidates for this method is limited. The development or discovery of more contrast agent choices deserves the attention of biochemists and chemists.

\section{Spectrum-based MCOCT}

OCT-based measurements can be processed to reveal spectroscopic tissue information at the cost of resolution degradation (73). Given that the spectroscopic information can reveal specific tissue properties, such as the scattering or absorption spectrum characteristics, image resolution compromise is often acceptable. An intuitive way to understand the tradeoff is by noting that OCT's axial resolution, as characterized by the coherence length $l_{c}=\ln (2)\left((2 / \pi)\left(\lambda^{2} / \Delta \lambda\right)\right)$, is directly proportional to the input light's spectrum bandwidth that we are willing to dedicate to the task of image resolution. Simplistically, we can obtain an $\mathrm{N}$ point tissue spectrum image set by segmenting the input light source spectrum into $\mathrm{N}$ parts and performing OCT imaging with each individual segment of the spectrum; the reduced spectrum of each segment that we are dedicating to OCT resolution implies that the resolution will be $\mathrm{N}$ times worse than if we had dedicated the entire spectrum for resolution.

Early spectroscopic OCT research demonstrated that such spectrum segmentation, through a more sophisticated approach of wavelet transformation, was indeed capable of providing additional tissue information. However, the spectral variation of absorption and scattering cannot be separated in such measurements because both terms contribute to the exponential attenuation of the measured OCT signal (see Eq. 2). This implies that it is difficult to uniquely identify the distribution of intrinsic biochemical distribution through their absorption spectral signature; the presence of morphological micron-sized structural variations in tissues can create significant scattering spectral variation in the optical regime.

This issue can be overcome to some extent by introducing appropriately chosen molecular contrast agents into the target sample; this approach forms the basis for spectrum-based MCOCT methods. In general, if the absorption contribution of the introduced contrast agent dominates over those attributable to the sample's intrinsic absorption and scattering, the acquired spectrum-based MCOCT measurements can be processed to reveal the distribution profile of the contrast agent within the sample.

There are several clear advantages associated with this type of MCOCT imaging methods. First, the methods interrogate for the presence of the molecules in a passive manner; there is no need to induce state changes in the molecules by optical excitation. The absence of a need for an excitation light field simplifies the imaging scheme and eliminates concerns of excessive incident light field intensity for clinical imaging applications. Second, as the methods will work for any molecular species with a well-defined absorption peak, the choices of possible molecular contrast candidates are significantly broader. 
Spectroscopic OCT for contrast imaging-The first reported adaptation of a spectrumbased OCT approach for MCOCT imaging can be found in Ref. (64). In this particular implementation, the OCT light source had a center wavelength of $780 \mathrm{~nm}$, and the contrast agent used is a near-IR dye (ADS7460, H. W. Sands Inc., Jupiter, FL) that has an absorption spectrum peak of $740 \mathrm{~nm}$ (see Fig. 6). The backreflected light from the sample was coherence gated, and the centroid of the backreflected light spectrum was calculated for each specific depth. Because the absorption peak of the dye is off-centered from the center wavelength of the OCT light source, the presence of the dye in the tissue will tend to skew the spectrum of the backscattered light component by absorbing part of the input light spectrum. The presence and concentration of the contrast agent above a given depth is indicated by the extent of the centriod's shift from the light source's center wavelength.

In the reported experiment, the imaging target was a celery stalk that was allowed to uptake a dye solution (concentration of $75 \mu M$ ). The target was imaged and processed to reveal the presence of the contrast agent within the vascular bundle of the celery stalk (see Fig. 6). The simplicity of the method is a big advantage. Although Xu et al. (64) reported that the spectrum centroid did not appear to be significantly shifted for the celery samples used in which no contrast agents were administered, Morgner et al. (73) reported a significant spectral centroid shift in the case of imaging through a Xenopus laevis tadpole. This can be attributed the spectral variation of the intrinsic scattering and absorption property of the sample. As mentioned earlier, it is a source of systematic error for spectrum-based MCOCT imaging methods and can potentially prevent the detection of the introduced contrast agent at low concentration.

Spectral triangulation MCOCT_-In an effort to compensate for the intrinsic scattering and absorption variation in the target sample, a different spectroscopic MCOCT method was recently developed (Ref. [66]) (see Fig. 7). This method is capable of compensating for the intrinsic first-order spectral variation in the target sample. Given that the average intrinsic scattering and absorption extinction coefficient of biological samples are dominantly monotonic functions of wavelength, we can expect the elimination of the intrinsic first-order spectral variation to significantly improve the quality of the MCOCT image thus collected.

The basic strategy of the method is as follows. The effective OCT probe spectrum is divided into three equal parts centered at evenly spaced wavelengths $\lambda_{1}, \lambda_{2}$ and $\lambda_{3}$. The center wavelength of the second segment, $\lambda_{2}$, is chosen to match with the absorption maximum peak of the contrast agent. OCT scans are then acquired for all three components of the spectrum$P_{\text {interference }}\left(\lambda_{1}, z\right), P_{\text {interference }}\left(\lambda_{2}, z\right)$ and $P_{\text {interference }}\left(\lambda_{3}, z\right)$. A corresponding intermediary quantity $U_{\text {spec } \Delta}(z)$, the number concentration of the contrast agent as a function of scan depth $z$ can be found from the three scans (66): 
$U(z)$

$$
\begin{aligned}
& =\frac{1}{\Delta \sigma} \ln \left(\frac{\sqrt{P_{\text {interference }}\left(\lambda_{1}, z\right) \times P_{\text {interference }}\left(\lambda_{3}, z\right)}}{P_{\text {interference }}\left(\lambda_{2}, z\right)}\right) \\
& =\frac{1}{\Delta \sigma} \ln \left(\mathrm{e}^{-\int_{0}^{z}\left[\frac{1}{2} \mu_{a, \text { contrast_agent }}\left(\lambda_{1}, z_{s}{ }^{\prime}\right)+\frac{1}{2} \mu_{a, \text { contrast_agent }}\left(\lambda_{3}, z_{s}{ }^{\prime}\right)-\mu_{a, \text { contrast_agent }}\left(\lambda_{2}, z_{s}{ }^{\prime}\right)\right] d z_{s}{ }^{\prime}}\right. \\
& \times \mathrm{e}^{-\int_{0}^{z}\left[\frac{1}{2} \mu_{a, \text { intrinsic }}\left(\lambda_{1}, z_{s}{ }^{\prime}\right)+\frac{1}{2} \mu_{a, \text { intrinsic }}\left(\lambda_{3}, z_{s}{ }^{\prime}\right)-\mu_{a, \text { intrinsic }}\left(\lambda_{2}, z_{s}{ }^{\prime}\right)\right] d z_{s}{ }^{\prime}} \\
& \left.\times \mathrm{e}^{-\int_{0}^{z}\left[\frac{1}{2} \mu_{s}\left(\lambda_{1}, z_{s}{ }^{\prime}\right)+\frac{1}{2} \mu_{s}\left(\lambda_{3}, z_{s}{ }^{\prime}\right)-\mu_{s}\left(\lambda_{2}, z_{s}{ }^{\prime}\right)\right] d z_{s}{ }^{\prime}} \frac{\sqrt{R\left(\lambda_{1}, z\right) \times R\left(\lambda_{3}, z\right)}}{R\left(\lambda_{2}, z\right)}\right) \\
& \approx \frac{-1}{\Delta \sigma} \int_{0}^{z}\left[\frac{1}{2} \mu_{a, \text { contrast_agent }}\left(\lambda_{1}, z_{s}{ }^{\prime}\right)+\frac{1}{2} \mu_{a, \text { contrast_agent }}\left(\lambda_{3}, z_{s}{ }^{\prime}\right)\right. \\
& \left.-\mu_{a, \text { contrast_agent }}\left(\lambda_{2}, z_{s}^{\prime}\right)\right] d z_{s}{ }^{\prime} \\
& =\int_{0}^{z} n\left(z_{s}^{\prime}\right) d z_{s}^{\prime},
\end{aligned}
$$

where $\Delta \sigma=\sigma\left(\lambda_{2}\right)-(1 / 2) \sigma\left(\lambda_{1}\right)-(1 / 2) \sigma\left(\lambda_{3}\right)$. The approximation in Eq. (12) is arrived by assuming that $\mu_{a \text {,intrinsic }}\left(\lambda, z_{s}^{\prime}\right), \mu_{s}\left(\lambda, z_{s}^{\prime}\right)$ and $R(\lambda, z)$ are either constant or linearizable with respect to wavelength. The method operates best when the dye molecule has a well define and sharp absorption maximum so that the contrast change $\left[(1 / 2) \mu_{a, \text { contrast_agent }}\left(\lambda_{1}, z_{s}{ }^{\prime}\right)+(1 / 2)\right.$ $\left.\mu_{a, \text { contrast_agent }}\left(\lambda_{3}, z_{s}^{\prime}\right)-\mu_{a, \text { contrast_agent }}\left(\lambda_{2}, z_{s}{ }^{\prime}\right)\right]$ is maximized.

As reported in Ref. (66), the researchers were able to detect the presence of an IR dye, ICG, at a concentration of $200 \mu M$ in a scattering medium consisting of $0.25 \%$ volume concentration suspension of $0.1 \mu \mathrm{m}$ microspheres to a depth of about $1.2 \mathrm{~mm}$ (see Fig. 7). The dye used has an absorption maximum at $790 \mathrm{~nm}$, and $\lambda_{1}, \lambda_{2}$ and $\lambda_{3}$ are chosen to be 760,795 and $830 \mathrm{~nm}$, respectively. The image acquisition process involved acquiring three sets of OCT images at the three wavelengths and tuning the center wavelength of the source laser to the appropriate wavelength between each acquisition.

The researchers also reported the ability to detect the contrast agent within a X. laevis tadpole in which the contrast agent at a concentration of $400 \mu M$ was injected into its gill structures.

\section{COHERENT EMISSION-BASED MCOCT METHODS}

At present, there are two reported MCOCT methods that fall within this category: second harmonic OCT (SH-OCT) (74-76) and CARS-based contrast OCT (named nonlinear interferometric vibrational imaging [NIVI] by its developers) (77). Both methods rely on using a molecular contrast agent within a target sample that can efficiently and coherently convert the incoming OCT probe light field into an emission that is detectable using interferometric approaches.

The detection method common to both methods involves interferometrically mixing the emission with a reference field that is generated from a reference generator. The input light to the sample and the reference generator must originate from the same light source. In the case of NIVI, the reference generator can be a cuvette of a highly concentrated solution of the same contrast agent; in the case of SH-OCT, the reference generator can simply be a second harmonic crystal. Because the emission from the sample and the reference generator are coherent and preserve a definite phase relationship with the original light source, they will be locked in phase with respect to each other. This implies that the mixing of the two light fields will interfere under the right conditions. 
These methods generally require the use of ultra short pulse light sources for two reasons. First, the nonlinear generation processes employed in the methods generally require high instantaneous light field intensities for efficient light conversion. Second, the associated broad spectral bandwidths of such light sources enable coherence-gated detection of the emission through OCT schemes for spatial resolution.

A distinct advantage of the methods in comparison with absorption-based MCOCT methods lies in the fact that the signal processing involved for coherent emission-based MCOCT methods is much simplified. The detected OCT image at the emission wavelength is a scattering attenuated distribution map of the contrast agents within the target sample. The paired baseline change image acquisition and processing approach required for absorption-based MCOCT methods are not required in this situation. A generalized expression for the coherent emissionbased MCOCT signal can be expressed as:

$$
\begin{aligned}
& P_{\text {interference }}\left(\lambda_{\text {emission }}, z\right) \approx 2 \sqrt{P_{\mathrm{Ro}} P_{S}\left(\lambda_{\text {emission }}, z\right)} \approx 2 \sqrt{P_{\mathrm{Ro}}} \\
& \times \sqrt{C\left(P_{\mathrm{So}}\left(\lambda_{\text {pump }, 1}, z\right) / A\left(\lambda_{\text {pump }, 1}, z\right), P_{\mathrm{So}}\left(\lambda_{\text {pump }, 2}, z\right) / A\left(\lambda_{\text {pump }, 2}, z\right), \ldots,\right) B(z)} \\
& \times n(z) A\left(\lambda_{\text {emission }}, z\right) I_{c} \mathrm{e}^{-\frac{1}{2} \int_{0}^{z_{0}}\left[\mu_{a}\left(\lambda_{\text {emission }}, z_{s}^{\prime}\right)+\mu_{s}\left(\lambda_{\text {emission }}, z_{s}{ }^{\prime}\right)\right] d z_{s}^{\prime}}
\end{aligned}
$$

where $P_{S}\left(\lambda_{\text {emission }}, z\right)$ is the collected emission from depth $z$ and $\lambda_{\text {emission }}$ is the emission wavelength. The last equation is an approximation in which we further simply the expression $P_{S}\left(\lambda_{\text {emission }}, z\right)$ into its basic components. $C\left(P_{\mathrm{So}}\left(\lambda_{\text {pump }, 1}, z\right) / A\left(\lambda_{\text {pump }, 1}, z\right), P_{\mathrm{So}}\left(\lambda_{\text {pump }, 2}, z\right) / A\right.$ $\left.\left(\lambda_{\text {pump }, 2}, z\right), \ldots\right)$ is the conversion efficiency of each molecule in changing the input optical powers into the emission optical power. It is to be expected to have a dependency on the localized incident pump intensities given by $P_{\mathrm{So}}\left(\lambda_{\text {pump }, i}, z\right) / A\left(\lambda_{\text {pump }, i}, z\right)$ (the intensities are, hereby, expressed on the basis of a simple top hat light field mode profile with an effective illumination area of $A\left(\lambda_{\text {pump }, i}, z\right)$; more realistic models can be applied in a straightforward manner). $B(z)$ is the fraction of the emitted photons from depth $z$ that fall within the collection numerical aperture of the OCT system, the exponential factor accounts for attenuation of the emitted light field on its passage out of the target sample. $n(z)$ is the number concentration of the contrast agent at location of interest, $A\left(\lambda_{\text {emission }}, z\right)$ is the effective area at sample depth $z$ from which the emission signal is collected and $l_{c}$ is the coherence length of the emission and represents the axial extent of the sample that will contribute to a single image voxel. As a whole, $n(z) A\left(\lambda_{\text {emission }}, z\right) l_{c}$ represents the total number of contrast agent molecules that will contribute to the signal corresponding to a single image voxel. The interference signal is a measure of the optical field strength associated with the emitted light field. Given that the coherent emission process is proportionally additive in optical field strength, we can expect $P_{S}\left(\lambda_{\text {emission }}, z\right)$ to be proportional to the square of the total number of signal contributing contrast agent molecule, $n(z) A\left(\lambda_{\text {emission }}, z\right) l_{c}$. Of course, the emission optical field strength depends to some extent on the spatial arrangement of the contrast agents as well. As such, the above approximation of modeling $P_{S}\left(\lambda_{\text {emission }}, z\right)$ as a linear function of $n(z)$ is a fair model, but it can be expected to fail in very specific conditions.

In comparison with the basic OCT signal equation (Eq. 2), we can see another important difference- the localized reflectivity of the sample, $R(\lambda, z)$, is no longer a necessary component of the signal consideration. In other words, as long as some components of the coherently generated emission are emitted in the backward direction, a positive MCOCT signal will be generated and detected, even if the medium does not scatter light at all. The localized reflectivity can potentially be a factor in the scenario where the nonlinear emission generation is forward directed and the sample's reflectivity has to be relied on to backscatter the light back into the collection aperture of the OCT system. 


\section{Second harmonic OCT}

The successful implementation of SH-OCT and the demonstration of the method's ability to acquire OCT type images was first reported in Ref. (74). Since then, several more implementations by other groups were reported (75-77).

Noninterferometric SHG microscopy (25-27), based on the concept of illuminating the target sample with a well focused pulsed light field and detecting the second harmonic emission from the focal spot with a PMT, has been applied in a biomedical context for examining tissue structures, interfaces, and molecular orientation. Because optical SHG generation process derives its emission based on the lowest nonlinear optical susceptibility, it is generally the most accessible and efficient nonlinear light generation process given the incident light intensity considerations.

SHG microscopy is especially useful for imaging collagen matrices and their orientation within biological samples. In principle, big and asymmetrical molecules with high nonlinear polarizability, such as bR $(78,79)$, are also possible molecular contrast candidates for $\mathrm{SH}$ microscopy-based imaging.

An SH-OCT imaging method can be expected to match the performance of SHG microscopy and surpass it in certain areas. Given that OCT-based detection schemes when implemented correctly can achieve shot noise detection limit, we can expect SH-OCT's signal to noise sensitivity to match that of SHG microscopy. In other words, for a given amount of SHG photons generated and collected, the ability of SH-OCT and SHG microscopy to detect them is comparable. With respect to the two method's ability to depth resolve the SH contribution, we can expect SH-OCT to outperform SH microscopy. In SHG microscopy, the depth discrimination derives from the fact that the SHG process is proportional to the square of the intensity, so most of the SHG light is generate at or near the focal spot. The extent of SHG generation falls off as a polynomial function as we move away from the focal point. In SHOCT, the coherence gating ability of OCT can be exploited to dramatically sharpen the resolution. For the case where the OCT light source has a Gaussian spectral profile, the coherence detection envelop will also be Gaussian in profile-a much sharper gating function than a polynomial function (80).

In the first reported implementation of SH-OCT, a $\beta$-barium borate second harmonic crystal was used to generate a reference second harmonic reference beam from an input $800 \mathrm{~nm}, 110$ fs pulse duration, $76 \mathrm{MHz}$ repetition rate Ti:Sapphire laser (see Fig. 8). The $50 \mathrm{~mW}$ of the laser light was focused on the sample to achieve a peak dower density of $\sim 3.2 \mathrm{GW} / \mathrm{cm}^{2}$ at the beam waist. The backscattered SHG was collected and interfered with the reference beam. The researchers were able to image and localize the collagen layers from their target sample consisting of collagen layers sandwiched between glass slides (see Fig. 8). Three other SHOCT implementations were reported by other research groups, one of which was of particular interest in that the published result reported on the imaging of the SHG generation from a biological target—salmon fish scales (see Fig. 8). Ref. (76) was also the first to report on the an SH-OCT measurement of the SHG's polarization as a way to discern the collagen matrix orientation in the target.

The expression for the conversion efficiency term in Eq. (13) for the SH-OCT case is given by:

$$
C\left(P_{\mathrm{So}}\left(\lambda_{\text {pump }}, z\right)\right)=C_{\mathrm{SHG}} P_{\mathrm{So}}\left(\lambda_{\text {pump }}, z\right)^{2} / A_{\text {pump }}(z)^{2}
$$

where $C_{\mathrm{SHG}}$ is the conversion efficiency of each molecule in changing the input fundamental light into second harmonic light. $C_{\mathrm{SHG}}$ is proportional to the square of the second-order 
susceptibility, $\chi^{2}(\omega)(81)$. This method has the potential of achieving excellent SNR as the efficiency of the SHG process improves as a square function of the input light intensity. The signal strength is critically dependent on the relative orientation of the molecules involved. Although the emission of similarly aligned molecules will emit in a constructive manner, emission from oppositely oriented molecules will actually interference destructively and reduce the effective emission. The equational form for the MCOCT signal is only valid for similarly aligned molecules.

\section{Nonlinear interferometric vibrational imaging}

NIVI makes use of the third-order nonlinear optical susceptibility of the target molecular contrast agent in a CARS scheme for contrast. The method was first reported in Ref. (82). A subsequent article on a different implementation of NIVI was published in Ref. (77).

The second implementation method is conceptually very similar to the demonstrated SH-OCT scheme reported above. For clarity, we shall first explain this second implementation method before we describe the first implementation method.

The method reported in Ref. (82) operates by combining two high-intensity light fields, Stokes (S) and pump (P), at wavelengths $\lambda_{S}$ and $\lambda_{P}$ on the targeted focal region (see Fig. 9). The method uses the third-order nonlinear optical susceptibility in a four-wave mixing process to generate an emission field, the Anti-Stokes (AS) field, at the optical frequency $\lambda_{\mathrm{AS}}$. The process is enhanced by the presence of a molecular contrast agent that has a vibrational mode in resonance with the Stokes field. The AS field will have a definite phase relationship with the A and P fields. By generating a similar AS field through a similar process within a cuvette filled with a high concentration of the same molecules, the sample's emission and this reference AS field can then be combined interferometrically to measure the distribution of the contrast agent within the sample.

The reported implementation used a modelock Ti:Sapphire, a regenerative amplifier, and an OPO to generate $250 \mathrm{kHz}$ repetition rate, $70 \mathrm{fs}$ light pulse train at 807 and $1072 \mathrm{~nm}$. Part of the two light beams was combined on a cuvette of benzene to generate an AS field at $647 \mathrm{~nm}$; this generated beam served as the reference beam. A similar cuvette of benzene served as the sample, and the combination of the Stokes and pump beam created a transmitted AS beam. The interference of the two beams constituted the demonstration that NIVI-based MCOCT is, in principle, possible.

The expression for the conversion efficiency term in Eq. (13) for NIVI is given by:

$$
\begin{aligned}
& C\left(P_{\text {So }}\left(\lambda_{\text {stokes }}, z\right), P_{\text {So }}\left(\lambda_{\text {pump }}, z\right)\right) \\
& =C_{\mathrm{NIVI}} P_{\mathrm{So}}\left(\lambda_{\text {Stokes }}, z\right) P_{\mathrm{So}}^{2}\left(\lambda_{\text {pump }}, z\right) \\
& =C_{\mathrm{NIVI}}(z) P_{\text {So }}\left(\lambda_{\text {Stokes }}, 0\right) P_{\text {So }}\left(\lambda_{\text {pump }}, 0\right)^{2} \\
& \times \mathrm{e}^{-\int_{0}^{z_{0}}\left[\mu_{a}\left(\lambda_{\text {Stokes }}, z_{s}{ }^{\prime}\right)+\mu_{s}\left(\lambda_{\text {Stokes }}, z_{s}{ }^{\prime}\right)\right] d z_{s}{ }^{\prime}-2 \int_{0}^{z_{0}}\left[\mu_{a}\left(\lambda_{\text {pump }}, z_{s}{ }^{\prime}\right)+\mu_{s}\left(\lambda_{\text {pump }}, z_{s}{ }^{\prime}\right)\right] d z_{s}{ }^{\prime}}
\end{aligned}
$$

where $C_{\mathrm{NIVI}}(z)$ is the conversion efficiency of each molecule in changing the input optical powers into the CARS emission. $C_{\mathrm{NIVI}}(z)$ is proportional to the square of the third-order susceptibility, $\chi^{(3)}(\omega)$.

The method has an interesting feature- - the wavelengths of the S, P and AS field can be arranged to be fairly close to each other by the judicious selection of S and P's wavelengths and an appropriate molecular vibrational mode. In the specific case of OCT-based imaging, 
this can be a significant advantage because it is highly desirable for all light fields involved to fall within the red or IR regime where tissue scattering tends to be low.

The first reported NIVI implementation method cleverly exploits this feature for another purpose - the elimination of the reference sample for generating a suitable AS reference field. In this particular method, the spectrum from a broadband pulsed light source (83) or a supercontinuum source (84) is segmented into P, S and AS components. Despite the fact that they are at different wavelengths, there is a deterministic phase relationship between the three components because they originate from the same phase-locked source.

In the reported experiment, the separation of the AS reference component from the other two components was achieved by splitting the input light with an appropriate dichroic mirror. The $\mathrm{P}$ and $\mathrm{S}$ light fields were used to probe and excite the target, and the generated AS field was then interferometrically mixed with the AS reference light field. This implementation method has the added advantage that by appropriately pulse shaping the input $\mathrm{P}$ and $\mathrm{S}$ fields, and time delaying the AS reference field, it is possible to measure the spectral variations in $\chi^{(3)}(\omega)$. This, in turn, implies that it is possible to identify specific chemical agents by their specific $\chi^{(3)}(\omega)$ spectral variation.

In terms of the sensitivity of the two coherent emission-based MCOCT methods we have discussed thus far, NIVI can be expected to generate weaker signals because it is a third-order optical susceptibility-based generation process.

Because CARS generation is enhanced by the presence of resonant vibrational modes, NIVI is more generally applicable in that it is possible to use it to obtain contrast distribution mapping of a far wider range of molecular species. In addition, the CARS signature of each molecular species is highly specific, which implies that NIVI has the potential capability to simultaneously image multiple molecular species within the sample.

There are significant challenges that will have to be overcome before NIVI-based MCOCT becomes practical. The light intensities levels used in the demonstration are too high to be acceptable for in vivo applications. In addition, the reported demonstration was performed in the transmission mode, presumably because the amount of CARS generated under the experimental conditions is low and it would be a more challenging task to detect the generally weaker, backward-propagating emission as opposed to the forward-propagating component. Given that epidetection of CARS signal has been recently reported in the context of CARS microscopy (Ref. [28]), researchers working on NIVI have expressed optimism that NIVIbased MCOCT will eventually be experimentally realized.

\section{SCATTERING-BASED MCOCT METHODS}

Thus far, there are two reported research efforts that can be categorized as scattering-based MCOCT methods. The contrast agents used in the methods are engineered microspheres (85) and magnetic particles (86).

Both methods rely on creating a sizable sample reflectivity, $R(\lambda, z)$, with the presence of the contrast agent and correlating the size of the measured reflectivity signal to the contrast agent's concentration. The presence of the scattering contrast agent above the specific depth of interest can potentially reduce the effective signal because the scatterers will contribute to the $\mu_{s}\left(\lambda, z_{s}^{\prime}\right)$ term as well. An optimal contrast agent for this application should therefore have a large backscatter signal component so that $R(\lambda, z)$ is maximized for a given $\mu_{s}\left(\lambda, z_{s}{ }^{\prime}\right)$ associated with the contrast agent. 
The use of scattering contrast agent has its associated advantages and disadvantages. One major advantage of the method is that the contrast signal will be significant because changing the reflectivity of the sample will lead to a very observable OCT signal change. A major disadvantage associated with the method is that the contrast agent must be functionalized to bind to the targeted chemical and biochemical species of interest to elicit contrast. Whereas all of the MCOCT methods that have been discussed, thus far, may potentially be applied to directly use the chemical species of interest as the contrast agent, such is not the case for this class of MCOCT methods-in general, a single molecule will not have a sufficiently large scattering cross-section to be directly detectable via this type of MCOCT methods.

\section{Engineered scatterer MCOCT}

Lee et al. (85) reported the first implementation of an MCOCT method on the basis of the use of an externally introduced scattering contrast agent. In this implementation, the contrast agent consisted of microspheres with an approximately $50 \mathrm{~nm}$ thick protein shell (see Fig. 10). These microspheres can range from 0.2 to $15 \mu \mathrm{m}$ in diameter, and nanoparticles and molecules can be incorporated into their cores or shells. The protein shells may also be directly functionalized to target the scatterers to specific regions of interest.

The researchers demonstrated the technique by fabricating gold-shelled, oil-filled microspheres with diameter of about $2 \mu \mathrm{m}$ and injecting the contrast agent into mice. The livers of the mice were then examined with OCT, and it was found that OCT images of livers from the treated mice are strongly scattering in comparison with those from a control. The localization of the microspheres in the liver was attributed to the phagocytosis of the microspheres by Kupffer cells in the liver.

The researchers reported that gold, melanin, carbon and oil can be incorporated into the microspheres to enhance the scattering. In the case where gold is used, the average scattering cross-section of the microspheres is about 0.56 times the physical cross-section.

The MCOCT signal associated with this method can be expressed as:

$$
\begin{aligned}
& P_{\text {interference }}(\lambda, z) \\
& \approx 2 \sqrt{P_{\mathrm{Ro}} P_{S}(\lambda, z)} \\
& =2 \sqrt{P_{\mathrm{Ro}} P_{\mathrm{So}}} \mathrm{e}^{-\int_{0}^{z_{[}\left[\mu_{a}\left(\lambda, z_{s}{ }^{\prime}\right)+\mu_{s}\left(\lambda, z_{s}{ }^{\prime}\right)\right] d z_{s}{ }^{\prime}} \sqrt{R(\lambda, z)}} \\
& =2 \sqrt{P_{\mathrm{Ro}} P_{\mathrm{So}}} \mathrm{e}^{-\int_{0}^{z}\left[\mu_{a}\left(\lambda, z_{s}{ }^{\prime}\right)+\mu_{s, \text { intrinsic }}\left(\lambda, z_{s}{ }^{\prime}\right)+\mu_{s, \text { contrast_agent }}\left(\lambda, z_{s}{ }^{\prime}\right)\right] d z_{s}{ }^{\prime}} \\
& \times \sqrt{R_{\text {intrinsic }}(\lambda, z)+R_{\text {contrast_agent }}}(\lambda, z)
\end{aligned}
$$

where $R(\lambda, z)$ can be expressed as a summation of the target's intrinsic reflectivity $R_{\text {intrinsic }}(\lambda, z)$ and the contrast agent-contributed reflectivity $R_{\text {contrast_agent }}(\lambda, z)$. The scattering extinction coefficient $\mu_{\mathrm{s}}\left(\lambda, \mathrm{z}_{\mathrm{s}}{ }^{\prime}\right)$ can also be expressed as a summation of the target's intrinsic scattering coefficient $\mu_{\mathrm{s}}$,intrinsic $\left(\lambda, \mathrm{z}_{\mathrm{s}}{ }^{\prime}\right)$ and the contrast agent-contributed scattering coefficient $\mu_{\mathrm{s}, \text { contrast_agent }}\left(\lambda, \mathrm{z}_{\mathrm{s}}{ }^{\prime}\right)$.

The equation can be simplified in two different limits. In the case where $R_{\text {intrinsic }}(\lambda, z)$ is much greater than $R_{\text {contrast_agent }}(\lambda, z)$, Eq. (16) reduces to (weak contrast scattering limit): 


$$
\begin{aligned}
& P_{\text {interference }}(\lambda, z)=2 \sqrt{P_{\mathrm{Ro}} P_{\mathrm{So}}} \mathrm{e}^{-\int_{0}^{z}\left[\mu_{a}\left(\lambda, z_{s}{ }^{\prime}\right)+\mu_{s}\left(\lambda, z_{s}{ }^{\prime}\right)\right] d z_{s}{ }^{\prime}} \\
& \times \sqrt{R_{\text {intrinsic }}(\lambda, z)+R} \text { contrast_agent }{ }^{(\lambda, z)} \\
& \approx 2 \sqrt{P_{\mathrm{Ro} P_{\mathrm{So}}}} \mathrm{e}^{-\int_{0}^{z_{0}}\left[\mu_{a}\left(\lambda, z_{s}{ }^{\prime}\right)+\mu_{s}\left(\lambda, z_{s}{ }^{\prime}\right)\right] d z_{s}{ }^{\prime}} \\
& \times\left(\sqrt{R_{\text {intrinsic }}(\lambda, z)}+\frac{1}{2} \frac{R_{\text {contrast_agent }}(\lambda, z)}{\sqrt{R_{\text {intrinsic }}(\lambda, z)}}\right) \\
& =2 \sqrt{P_{\mathrm{Ro}} P_{\mathrm{So}}} \mathrm{e}^{-\int_{0}^{z_{0}}\left[\mu_{a}\left(\lambda, z_{s}{ }^{\prime}\right)+\mu_{s}\left(\lambda, z_{s}{ }^{\prime}\right)\right] d z_{s}{ }^{\prime}} \\
& \times\left(\sqrt{R_{\text {intrinsic }}(\lambda, z)}+\frac{1}{2} \frac{n(z) I_{c} \sigma_{\text {backscatter }}{ }^{(\lambda)}}{\sqrt{R_{\text {intrinsic }}(\lambda, z)}}\right)
\end{aligned}
$$

where $R_{\text {contrast_agent }}(\lambda, z)$ can be expressed as $n(z) l_{c} \sigma_{\text {backscatter }}(\lambda) . n(z)$ is the concentration of the scatterers at depth $\mathrm{z}, l_{c}$ is the coherence length of the OCT system and it defines the axial extent of the target's volume that will contribute to a single OCT image voxel and $\sigma_{\text {backscatter }}(\lambda)$ is the backscattering cross-section of a scatterer. This equation is an approximation because it ignores the possible speckle character of the net reflectivity from the depth of interest. In the case where $R_{\text {intrinsic }}(\lambda, z)$ is much smaller than $R_{\text {contrast_agent }}(\lambda, z)$, Eq. (16) can be simplified as (strong contrast scattering limit):

$$
\begin{aligned}
& P_{\text {interference }}(\lambda, z)=2 \sqrt{P_{\mathrm{Ro}} P_{\mathrm{So}}} \mathrm{e}^{-\int_{0}^{z}\left[\mu_{a}\left(\lambda, z_{s}{ }^{\prime}\right)+\mu_{s}\left(\lambda, z_{s}{ }^{\prime}\right)\right] d z_{s}{ }^{\prime}} \\
& \times \sqrt{R_{\text {intrinsic }}(\lambda, z)+R_{\text {contrast_agent }}(\lambda, z)} \\
& \approx 2 \sqrt{P_{\mathrm{Ro}} P_{\mathrm{So}}} \mathrm{e}^{-\int_{0}^{z_{0}}\left[\mu_{a}\left(\lambda, z_{s}{ }^{\prime}\right)+\mu_{s}\left(\lambda, z_{s}{ }^{\prime}\right)\right] d z_{s}{ }^{\prime}} \\
& \times\left(\sqrt{R_{\text {contrast_agent }}(\lambda, z)}\right)
\end{aligned}
$$

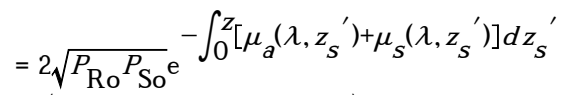

$$
\begin{aligned}
& \times\left(\sqrt{n(z) I_{c} \sigma_{\text {backscatter }}(\lambda)}\right)
\end{aligned}
$$

The reported experiments were performed in the later limit. However, the weak contrast scattering limit is of relevance in determining the ultimate sensitivity limit of the method. The topic will be discussed in Signal strength.

One possible disadvantage of the above method is that the scattering change due to the contrast agent is indistinguishable from the intrinsic scattering contribution from the target. In order for the scattering profile to be a direct map of the contrast agent distribution, the scattering contribution from the contrast agent must be sufficiently large to make the intrinsic scattering contribution negligible.

\section{Magnetically modulated MCOCT}

This second method of scattering-based MCOCT relies instead on a scattering contribution from the contrast agent that is distinguishable from the intrinsic target scattering. The contrast agent used is composed of ferromagnetic hematite particles.

In a recent reported demonstration of this MCOCT method (86), the contrast agent was taken up by a cell culture of macrophages via phagocytosis (see Fig. 11). The cell culture was then imaged with an OCT system in the presence of an oscillating magnetic field (about $300 \mathrm{G}$ and 
at a frequency of $1 \mathrm{kHz}$ ). By locking into the image signal change that was occurring at the oscillation frequency of the magnetic field, the distribution of the magnetic particles within the sample can be found.

There are two possible mechanisms by which the motion of the magnetic particles in the changing magnetic field can create the scattering signal change. The magnetic particles can simply be shifting in and out of the OCT probe beam path. In the case where the magnetic particles are nonuniform in shape, it is also possible to create a scattering signal change if the particles simply rotate in place and present a varying scattering cross-section during their rotation.

An expression for the OCT signal in this case is given by:

$$
\begin{aligned}
& P_{\text {interference }}(\lambda, z) \\
& =2 \sqrt{P_{\mathrm{Ro}} P_{\mathrm{So}}} \mathrm{e}^{-\int_{0}^{z}\left[\mu_{a}\left(\lambda, z_{s}{ }^{\prime}\right)+\mu_{s}\left(\lambda, z_{s}{ }^{\prime}\right)\right] d z_{s}{ }^{\prime}} \\
& \times \sqrt{R_{\text {intrinsic }}(\lambda, z)+R_{\text {contrast_agent }}(\lambda, z)+\Delta R_{\text {contrast_agent }}(\lambda, z) \cos (\Omega t)+\cdots} \\
& \approx 2 \sqrt{P_{\mathrm{Ro}} P_{\mathrm{So}}} \mathrm{e}^{-\int_{0}^{z_{0}\left[\mu_{a}\left(\lambda, z_{s}{ }^{\prime}\right)+\mu_{s}\left(\lambda, z_{s}{ }^{\prime}\right)\right] d z_{s}{ }^{\prime}} \ldots} \\
& \times\left(\sqrt{R_{\text {intrinsic }}(\lambda, z)+R_{\text {contrast_agent }}(\lambda, z)}\right. \\
& \left.+\frac{1}{2} \frac{\Delta R_{\text {contrast_agent }}(\lambda, z) \cos (\Omega t)}{\sqrt{R_{\text {intrinsic }}(\lambda, z)+R_{\text {contrast_agent }}(\lambda, z)}}+\cdots\right) \\
& \approx 2 \sqrt{P_{\mathrm{Ro}} P_{\mathrm{So}}} \mathrm{e}^{-\int_{0}^{z_{0}}\left[\mu_{a}\left(\lambda, z_{s}{ }^{\prime}\right)+\mu_{s}\left(\lambda, z_{s}{ }^{\prime}\right)\right] d z_{s}{ }^{\prime}} \ldots \\
& \times\left(\sqrt{R_{\text {intrinsic }}(\lambda, z)+R_{\text {contrast_agent }}(\lambda, z)}\right. \\
& \left.+\frac{1}{2} \frac{n(z) I_{c} \sigma_{\text {backmatter }}(\lambda) O(\Omega) \cos (\Omega t)}{\sqrt{R_{\text {intrinsic }}(\lambda, z)+n(z) I_{c} \sigma_{\text {backmatter }}(\lambda)}}+\cdots\right)
\end{aligned}
$$

The definitions of most of the terms are the same as those from Eq. (16). The perturbative term $\Delta R_{\text {contrast_agent }}(\lambda, z) \cos (\Omega t)$ is given by the reflectivity changes that occur at the oscillating magnetic field's frequency $\Omega$. There may be higher order perturbative terms, but they can be expected to be smaller in magnitude. As with the previous equation, this equation is an approximation because it ignores the possible speckle character of the net reflectivity from the depth of interest. $\Delta R_{\text {contrast_agent }}(\lambda, z)$ can in turn be expressed as $R_{\text {contrast_agent }} O(\Omega)$ or $n(z)$ $l_{c} \sigma_{\text {backscatter }}(\lambda) O(\Omega)$, where $O(\Omega)$ is the fractional reflectivity that is modulating at frequency $\Omega$.

The contrast signal can be obtained by finding the frequency component of the OCT interference signal at the oscillating magnetic field's frequency $\Omega$ :

$$
\begin{aligned}
& P_{\text {contrast }}(\lambda, z)=\left|\int P_{\text {interference }}(\lambda, z) \mathrm{e}^{-i \Omega t} d t\right| \\
& \approx 2 \sqrt{P_{\mathrm{Ro}^{P_{\mathrm{So}}}}}-\int_{0}^{z}\left[\mu_{a}\left(\lambda, x_{s}{ }^{\prime}\right)+\mu_{s}\left(\lambda, x_{s}{ }^{\prime}\right)\right] d x_{s}{ }^{\prime} \\
& \times\left(\frac{1}{4} \frac{n(z) I_{c} \sigma_{\text {backscatter }}(\lambda) O(\Omega)}{\sqrt{R_{\text {intrinsic }}(\lambda, z)+n(z) I_{c} \sigma_{\text {backscatter }}(\lambda)}}\right)
\end{aligned}
$$


We can simplify the above equation in two different limits. If the intrinsic reflectivity contribution is much larger than that associated with the contrast agent, the above equation reduces to (weak contrast scattering limit):

$$
\begin{aligned}
& P_{\text {contrast }}(\lambda, z) \approx 2 \sqrt{P_{\mathrm{Ro}} P_{\mathrm{So}}} \mathrm{e}^{-\int_{0}^{z}\left[\mu_{a}\left(\lambda, x_{s}{ }^{\prime}\right)+\mu_{s}\left(\lambda, x_{s}{ }^{\prime}\right)\right] d x_{s}{ }^{\prime}} \\
& \times\left(\frac{1}{4} \frac{n(z) I_{c} \sigma_{\text {backscatter }}(\lambda) O(\Omega)}{\sqrt{R_{\text {intrinsic }}(\lambda, z)}}\right) .
\end{aligned}
$$

On the other hand, if the opposite is true, the equation reduces to (strong contrast scattering limit):

$$
\begin{aligned}
& P_{\text {contrast }}(\lambda, z) \approx 2 \sqrt{P_{\mathrm{Ro}} P_{\mathrm{So}}}-\int_{0}^{z}\left[\mu_{a}\left(\lambda, x_{s}{ }^{\prime}\right)+\mu_{s}\left(\lambda, x_{s}{ }^{\prime}\right)\right] d x_{s}{ }^{\prime} \\
& \times\left(\frac{1}{4} \sqrt{n(z) l_{c} \sigma_{\text {backscatter }}(\lambda)} O(\Omega)\right) .
\end{aligned}
$$

In summary, this MCOCT method is particularly promising because the measured contrast signal profile is a direct map of the contrast agent distribution in the sample. Furthermore, the method introduces another possible avenue of information extraction through the magnetic field used in the method. For example, it is possible to interrogate the stiffness of the medium that the particles are embedded in by altering the strength of the magnetic field.

\section{DISCUSSION}

Having discussed the various MCOCT methods that have been reported thus far, we shall now compare the existing and anticipated performance of the methods. Of primary concern to the eventual adaptation of the methods for biomedical applications are the following issues: contrast agent choice and range, contrast agent toxicity, the required light fluence level and signal sensitivity.

\section{Contrast agent choice and range}

An ideal MCOCT imaging modality should be able to image a wide range of contrast agents and be able to identify each contrast agent uniquely. The second ability is extremely helpful when the simultaneous tracking of two or more chemical species within the sample is desired; it will then be possible to functionalize different contrast agents to bind to each chemical species and provide distinguishable contrast signals. Alternatively, if the chemical species of interest are good contrast agents themselves, it is very desirable for an MCOCT method to be able to distinguish them.

Of the methods that we have discussed, NIVI has the greatest potential of approaching this twin ideal. As mentioned earlier, the associated CARS signal from different molecular species can be highly specific. The uniqueness can potentially allow NIVI to track the presence of different contrast agents within the same sample simultaneously. Moreover, the range of molecules with accessible vibrational modes is extensive; NIVI-based imaging can be expected to find a host of suitable molecular contrast agents for its applications. The use of NIVI-based imaging offers another intriguing possibility - the opportunity to track different populations of the same molecular species. CARS-based microscopy experiments have demonstrated that, by using different atomic isotopes in the synthesis of specific chemical compounds, it is possible to obtain molecules of the same chemical species that are differentiated on the basis of the isotope-induced CARS spectral shifts (87-89). For example, benzene that contains deuterium in place of normal hydrogen atoms should exhibit a shifted and distinct CARS 
signature. NIVI can potentially exploit this advantage to track the dynamics of the same contrast agent introduced into an organism at two or more spatially distinct locations.

Spectrum-based MCOCT methods offer the next widest possible molecular contrast agent choices. The simplicity of the technique and the fact that they do not require any specific optically induced state changes in the molecule for their operation imply that any molecular species that has a distinct absorption peak in the OCT imaging wavelength range is a suitable contrast agent candidate. Of the possible dye choices, ICG is a particularly favorable contrast agent choice because it is FDA approved for human use and its absorption peak is in the nearIR regime. The range of clinically suitable contrast agent choices can be expected to increase as the techniques mature in their implementations.

PPOCT can be expected to have a fairly wide range of molecular contrast agent choices. Finding molecular species that have a suitable triplet-triplet absorption in the OCT imaging wavelength range is fairly straightforward. The contrast agent choice used in the first PPOCT demonstration, methylene blue, is a particularly suitable one because it is an FDA-approved dye. A good molecular contrast agent for PPOCT should have a longer triplet relaxation lifetime so that the pump light intensity requirement is lower and the time window in which the molecules can be imaged in their shelved triplet state is longer.

Pump-suppression MCOCT has a more restrictive range of possible molecular contrast agent choices. The optochemical interaction involved, an optical switching of the molecules from one stable state to another stable state with a different absorption spectrum, is a fairly exotic interaction. Aside from the two possible candidates already mentioned, $b R$ and phyA, the wildtype and a mutant strain (S65T) of GFP (90-92) can potentially serve in the role of the contrast agent. The possible application of the technique with GFP is especially intriguing because GFP is already extensively used as an endogenously expressed fluorescent marker in biomedical research. However, it is to be noted that GFP has only been demonstrated to have two alternate optically switchable stable states with distinct fluorescence emission capability. To our knowledge, it has not been shown that the two states have distinguishable absorption spectra. Optically switchable chemical species have been studied for a myriad range of other applications. For example, there is extensive research into photochromism - the light-induced reversible transformation of chemical species between two isomers having different absorption spectra (93-95). Given the specific advantages that pump-suppression MCOCT possess, research into the development of new chemical species that can function as contrast agents for this method is highly desired.

SH-OCT's contrast agent species have to possess high intrinsic $C_{\mathrm{SHG}}$ coefficients. In formal terms, this implies that the agents must have strong hyperpolarizablity characteristics; interested readers are encouraged to refer to Ref. (81) for a deeper understanding of the topic. Some of the potential contrast agent candidates are structured collagen matrix, bR (69), and transmembrane-bindable contrast agents, such as styrl dye (96-99). The unique sensitivity of SH-OCT, in comparison with the other MCOCT methods, to the orientation of the molecules with respect to each other, is both a disadvantage and an advantage. It is a disadvantage because the introduction of a molecular contrast agent into the target sample for imaging is unlikely to yield a significant SHG signal, unless the molecules have a way to automatically align themselves within the target itself. It is potentially an advantage because the possible interaction of the chosen molecules with specific tissue types that leads to the self-alignment of the molecules will enhance the SHG generation and allows for selective imaging of tissue regions in which such self-alignment may have occurred. This can potentially be a unique MCOCT approach for interrogating tissue structure and chemical interaction. A related SH microscopy example of such a means for imaging structures can be found in the use of styrl (96-99) for SH imaging of cell membrane and profiling membrane dynamics, whereby the attachment of 
styrl onto the cell membrane in a self-aligned manner leads to a large SHG signal in comparison with free-floating styrl in a solution.

The scattering-based MCOCT methods are restricted in their contrast agent choices. Although, the engineered scatterer MCOCT method demonstration showed that different scatterer types can be fabricated by varying the material used. It is quite possible to engineer scatterer types to have different spectral scattering variation from each other. In such a manner, it may be possible to distinguish different scatterer types within a target by performing a spectral analysis of the acquired MCOCT images.

\section{Toxicity considerations}

The toxicity issue associated with the MCOCT imaging has to be assessed on two fronts: (1) the intrinsic toxicity of the contrast agents involved and (2) the toxicity of the side products that are generated by the optochemical interactions involved in the imaging process.

The intrinsic toxicity of the contrast agents involved in NIVI MCOCT imaging can be expected to be low. Because the range of potential CARS contrast agent is very wide, it should be possible to find numerous chemical species that are nontoxic to function as contrast agents.

Biochemicals, such as various lipid types, can serve as nontoxic contrast agents for NIVI. Toxicity of the chemical agents involved should also be a nonissue for pump-probe-based and spectrum-based MCOCT by reason of the relatively large range of contrast agents that they can use. The dyes that have already been used in the methods, methylene blue for pump-probe MCOCT and ICG for spectral triangulation MCOCT, are FDA approved for clinical applications. More nontoxic dye species can be expected to be found suitable for use with both techniques as research progresses. SH-OCT is an indeterminate case for this consideration because the sort of externally introducible chemical contrast agent suitable for use with the method is still unclear. The intrinsic toxicity of the contrast agents is expected to be a major issue for pump-suppression MCOCT. The contrast agent candidates that have been uncovered thus far are both proteins. The introduction of foreign proteins into an animal or human will generally elicit antigenic responses; these responses can potentially disrupt normal biological functions and skew any chemical distribution mapping that MCOCT aims to acquire. One possible way to overcome this problem is to genetically alter the animal to express these proteins endogenously. In such a situation, the toxicity of the contrast agent will be minimal. The contrast agent used in the engineered scatterer MCOCT method is relatively nontoxic. The use of gold particles in human subjects has been approved by FDA as a dietary supplement. Likewise, the magnetic hematite particles used in the magnetically modulated MCOCT method is presumed harmless at low dosage.

In addition to our consideration of the intrinsic toxicity of the contrast agent, we need to be mindful of the toxicity of the various chemical by-products that result from the optochemical interactions between the contrast agents and the excitation or probe light field. Of primary concern in this respect is the possibility that the light fluence level used in the schemes will result in the excessive formation of singlet oxygen (100) and free radicals that are destructive to the tissue involved. Singlet oxygen and the associated free radicals can be formed when molecules are forced into the triplet states, which necessitate a spin-forbidden relaxation back into the ground state. This relaxation is often facilitated by a spin exchange with oxygen and the creation of singlet oxygen.

The method for pump PPOCT as reported in Ref. (62) specifically shelves molecules into the triplet states. As such, PPOCT can be expected to generate a significant amount of singlet oxygen during the imaging process. NIVI and SH-OCT, by reason of their high instantaneous light intensity requirement, can also be expected to generate a significant amount of singlet oxygen. The generation is simply because of the incidental excitation of molecules into the 
triplet state under the intense light field. Pump-suppression MCOCT, spectrum-based MCOCT and the scattering-based MCOCT should not generate any significant amount of singlet oxygen because their probe light intensity requirements are very low.

\section{Light fluence considerations}

In addition to the toxicity issues addressed in the above section, we will also need to address the issue of possible direct photoinduced damage to the target before any of the MCOCT methods can be applied for biomedical research or clinical use. Generally, there are three intensity limits that we need to consider. The first limit is set by the threshold of irreversible cell damage and is applicable in the context of biomedical research that do not involve human subjects. The other two limits are set by the clinical safety guidelines issued by ANSI for the use of lasers on human subjects (37). In comparison with the photodamage limit, the ANSI limits are more stringent.

This section will discuss in brief the three light intensity limits, evaluate the maximum light intensities that were used in the various reported applications of the MCOCT methods and compare them with the three limits.

Damage threshold-The issue of photodamage by high-intensity illumination has been extensively studied in the context of two-photon fluorescence microscopy $(101,102)$. The damage mechanism associated with high instantaneous intensity light illumination has been conjectured to be due to the destructive intracellular plasma formation or the destructive singlet oxygen formation (or both) and indirect DNA damage. A convenient assessment benchmark for cell damage is the loss of the ability to reproduce.

One report (101) on the issue of photodamage in biological targets measured the threshold for photodamage to be at an instantaneous intensity of $690 \mathrm{GW} / \mathrm{cm}^{2}$ for light of wavelength 780 $\mathrm{nm}$ and pulse duration of $240 \mathrm{fs}$. The same report also established that the photodamage threshold is dependant on the pulse duration. For a shorter pulse of the same energy and wavelength, the damage threshold intensity increases as the inverse square root function of the pulse duration.

ANSI limits-The ANSI suggested guidelines for the use of lasers on human subjects can be found in Ref. (37). The imposed limits are generally given in terms of the maximum permissible exposure fluence (dimension of $\mathrm{J} / \mathrm{cm}^{2}$ ).

The first limit sets a maximum permissible fluence that a given location can be exposed to a continuous wave or pulse light field. The input fluence, as given by the product of the average light intensity with the dwell time, must be less than the limit. The dwell time, or exposure time, is simply defined as the duration for which a given target location is exposed to the light field. In a typical OCT imaging system that is capable of $10 \mathrm{kHz}$ A-scan rate, the dwell time will be equal to $0.1 \mathrm{~ms}$.

The second limit applies only to pulsed light sources. This limit sets a maximum fluence that a given location can be exposed to a single light pulse. In other words, the fluence of a single light pulse incident on the target, as given approximately by the product of the maximum instantaneous pulse intensity and the pulse duration, must be less than the limit.

Light exposure analysis of existing implementations of MCOCT methods-The following analysis estimates the maximum instantaneous and average intensities involved in the various MCOCT schemes that have so far been reported. We have taken the liberty of providing reasonable estimates of the experimental parameters when such are missing from the references. We can reasonably expect the calculated values to be within an order of 
magnitude of the actual values. We note that the maximum intensity involved in an experiment might not be that of the probe beam but may instead be that of the pump beam involved. We take only the highest intensity involved into consideration in this analysis.

Unlike in the NIVI and SH-OCT schemes, the contrast signal for the PPOCT experiment is not a direct function of the pump pulse duration. The experiment can be expected to give approximately the same contrast signal with the same energy per pulse but a much longer pump pulse duration (up to the triplet state relaxation lifetime of about $2 \mu \mathrm{s}$ ). Our analysis accounts for this by setting the pulse parameters for the pump-probe experiment to correspond to this longer duration. The same reasoning applies for the pump-suppression MCOCT experiment, the spectrum-based OCT experiments and both the scattering-based MCOCT experiments; all these experiments can be performed with continuous wave light beams.

The ANSI limits are calculated in two ways. We base our calculations on the ANSI guidelines for skin exposure. (1) Per dwell time limit. This limit is calculated for the average light intensity allowed on the target with the assumption that we are scanning the light beam at a sufficient speed such that the light is incident on any given location on the sample for a dwell time of 0.1 ms. This exposure time can be achieved in an MCOCT imaging system that is capable of acquiring A-scans at a rate of $10 \mathrm{kHz}$. (2) Per pulse limit. This limit is computed for the maximum instantaneous pulse intensity that is allowed on the target. ANSI exposure guideline does not cover the exposure time range for femtosecond pulses on skin, as such we were only able to calculate the exposure limit for the pump-probe scheme where the light pulse duration can be of the order of microseconds.

Finally, the reference used as our computation basis for photodamage limit was a study based on short-pulse laser systems. Its result cannot be expected to extend well into the continuous wave regime, as such we did not attempt to calculate photodamage thresholds for the MCOCT methods that can use continuous wave laser sources.

Table 1 clearly illustrates the very significant maximum instantaneous intensity requirement differences between the techniques. The light intensities involved in spectrum-based MCOCT, pump-suppression MCOCT, and scattering-based MCOCT methods are all low. PPOCT requires fairly high instantaneous pump intensity for its operation. Because the operation of SH-OCT and NIVI involves nonlinear optical generation processes, the required instantaneous light intensities are understandably high. Interestingly, almost all of the MCOCT methods are operating well within the photodamage threshold limit. The exception, NIVI, exceeds the threshold slightly. All of the absorption-based and scattering-based MCOCT methods are operating within the ANSI guideline. SH-OCT and NIVI methods exceed the limit by an order of magnitude.

The fact that all of the absorption-based methods are operating within the two limits is a positive indication that this class of MCOCT imaging methods can be reasonably expected to be applicable in a clinical setting eventually. The present implementations of coherent emissionbased MCOCT methods do exceed the ANSI limit dramatically. This issue is of particular concern for NIVI, the reported implementation was a simplified demonstration of the operating principle in which the CARS signal is collected in the transmission mode. An implementation of the method to detect the much weaker CARS signal in a backscatter geometry can be expected to require far higher light fluence exposure.

\section{Signal strength}

The sensitivities of the various MCOCT methods are of perhaps the greatest importance and relevance to the eventual deployment of the methods for biomedical or clinical applications. In this section, we propose a simple model by which the signal sensitivity of the various 
MCOCT methods can be analyzed and compared. We will also examine in brief the key signal sensitivity difference between the various MCOCT methods. The subject of speckle-induced errors in absorption-based MCOCT methods and the approaches for minimizing such errors will also be discussed. The subject of coherent light attenuation during its passage into and out of a target sample, and the differing degree to which the signal sensitivity of the various MCOCT methods are degraded, will also be discussed. This section will hopefully serve as a guide for assessing new MCOCT methods or new contrast agent choices.

The fundamental limit on contrast agent sensitivity can ultimately be traced to the shot noise limit of the underlying OCT system. Assuming that a given MCOCT system is shot noise limited, the contrast agent sensitivity limit can be estimated by considering the target sample shown in Fig. 12. We assume the sample consists of a medium of known scattering and intrinsic absorption extinction coefficients. We next assume that the sample contains a thin layer of contrast agent at a depth of $z$ and the thickness of the layer is $\Delta L$. For simplicity, we assume that $\Delta L$ is shorter than the coherence length $l_{c}$ of the OCT system used.

The analysis for absorption-based, coherent emission-based and scattering-based MCOCT methods are slightly different. We shall examine each separately and compare the results thereafter.

For absorption-based MCOCT methods, the ability of the methods to detect the presence of the layer containing the contrast agent requires that the difference in the detected OCT signals from the region just below the layer containing the contrast agent is sufficiently larger than the shot noise term associated with the detection system. If our measurement time is given by time $T$, the detection limit can be mathematically expressed as:

$$
\begin{aligned}
& \left|\frac{1}{2} P_{\text {interference, baseline }}(\lambda, z)-\frac{1}{2} P_{\text {interference, change }}(\lambda, z)\right|(\varepsilon T / \mathrm{h} v) \\
& >\sqrt{\varepsilon P_{\mathrm{Ro}} T / \mathrm{h} v} \\
& \times \sqrt{P_{\mathrm{Ro}} P_{\mathrm{So}}} \sqrt{R(\lambda, z)} e^{-\int_{0}^{z_{0}}\left[\mu_{a, \text { intrinsic }}\left(\lambda, z_{s}{ }^{\prime}\right)+\mu_{a, \text { contrast_agent_baseline }}\left(\lambda, z_{s}{ }^{\prime}\right)+\mu_{s}\left(\lambda, z_{s}{ }^{\prime}\right)\right] d z_{s}{ }^{\prime}} \\
& \times\left(\left|\Delta \mu_{a, \text { contrast_agent }}(\lambda, z)\right| \Delta L\right)(\varepsilon T / \mathrm{h} v)>\sqrt{\varepsilon P_{\mathrm{Ro}} T / \mathrm{h} v} \\
& \times \sqrt{P_{\mathrm{Ro}} P_{\mathrm{So}}} \sqrt{R(\lambda, z)} e^{-\int_{0}^{z}\left[\mu_{a, \text { intrinsic }}\left(\lambda, z_{s}{ }^{\prime}\right)+\mu_{a, \text { contrast_agent_baseline }}\left(\lambda, z_{s}{ }^{\prime}\right)+\mu_{s}\left(\lambda, z_{s}{ }^{\prime}\right)\right] d z_{s}{ }^{\prime}} \\
& \times(|\Delta n(z) \Delta \sigma| \Delta L)(\varepsilon T / \mathrm{h} v)>\sqrt{\varepsilon P_{\mathrm{Ro}} T / \mathrm{h} v} \text {. }
\end{aligned}
$$

The term on the right hand side of the equation is the shot noise term, whereas the term on the right is the difference signal. The sensitivity limit of the detection is reached when the two terms are equal. The inequality is evaluated in the thin contrast agent layer slice limit: | $\Delta \mu_{a \text {, contrast_agent }}(\lambda, z) \mid \Delta L \ll 1$.

We can reexpress the above equation as an SNR equation that relates the MCOCT's SNR in terms of the SNR of the OCT system:

$$
\begin{aligned}
& \mathrm{SNR}_{\mathrm{MCOCT}}=\left(\varepsilon P_{\mathrm{So}} T / \mathrm{h} v\right) \\
& \times\left(R(z) \mathrm{e}^{-2 \int_{0}^{z}\left[\mu_{a, \text { intrinsic }}\left(\lambda, z_{s}{ }^{\prime}\right)+\mu_{a, \text { contrast_agent_baseline }}\left(\lambda, z_{s}{ }^{\prime}\right)+\mu_{s}\left(\lambda, z_{s}{ }^{\prime}\right)\right] d z_{s}{ }^{\prime}}\right) \\
& \times(|\Delta n(z) \Delta \sigma| \Delta L)^{2}
\end{aligned}
$$

We simply divided Eq. (23) by the shot noise term and derived a dimensionless ratio. The first term of the ratio is equal to the SNR of the OCT system. The second term is equivalent to the 
fraction of input photons backscattered from the depth of $z$ and collected by the OCT detection system. In the absence of the third term, equating the ratio to unity will allow us to calculate the maximum penetration depth of the OCT system (given by the $z$ value found in the equation). The third term of the equation characterizes the sensitivity of the particular OCT system to the contrast agent. This sensitivity is naturally dependent on the depth of the absorptive layer; the deeper the layer is (larger $z$ value), the harder it is to detect it because the second term will be a smaller multiplicative factor for larger $z$ value. We can find the concentration that the contrast agent will have to be in order for the layer to be detectable by equating Eq. (24) to unity and inserting the depth of interest, $z$, into the equation.

The MCOCT detection sensitivity for each of the absorption-based methods can be found by substituting the appropriate expression for $\Delta n(z)$ and $\Delta \sigma$ into Eq. (24). The result is shown in Table 1.

The SNR associated with coherent emission-based MCOCT methods can be similarly analyzed through the same simple model. In this class of MCOCT methods, the detection limit is determined by:

$$
\begin{aligned}
& \frac{1}{2} P_{\text {interference }}(\lambda, z)\left(\lambda_{\text {emission }}, z\right)(\varepsilon T / \mathrm{h} v)>\sqrt{\varepsilon P_{\mathrm{Ro}} T / \mathrm{h} v} \sqrt{P_{\mathrm{Ro}}} \\
& \times \sqrt{C\left(P_{\mathrm{So}}\left(\lambda_{\text {pump, } 1}, z\right) / A\left(\lambda_{\text {pump, }}, z\right),\left(P_{\mathrm{So}}\left(\lambda_{\text {pump, }}, z\right) / A\left(\lambda_{\text {pump }, 2}, z\right), \ldots\right) B(z)\right.} \\
& \times n(z) A\left(\lambda_{\text {emission }}, z\right) \Delta L\left(e^{-\frac{1}{2} \int_{0}^{z}\left[\mu_{a}\left(\lambda_{\text {emission }}, z_{s}{ }^{\prime}\right)+\mu_{s}\left(\lambda_{\text {emission }}, z_{s}{ }^{\prime}\right)\right]}(\varepsilon T / \mathrm{h} v)\right. \\
& >\sqrt{\varepsilon P_{\mathrm{Ro}} T / \mathrm{h} v}
\end{aligned}
$$

The corresponding SNR equation is given by:

$$
\begin{aligned}
& \operatorname{SNR}_{\mathrm{MCOCT}}=(\varepsilon T / \mathrm{h} v) \times C\left(P_{\mathrm{So}}\left(\lambda_{\text {pump }, 1}, z\right) / A\left(\lambda_{\text {pump }, 1}, z\right)\right. \\
& \left.\times P_{\mathrm{So}}\left(\lambda_{\mathrm{pump}, 2}, z\right) / A\left(\lambda_{\mathrm{pump}, 2}, z\right), \ldots\right) \times B(z) \\
& \times\left(n(z) A\left(\lambda_{\text {emission }}, z\right) \Delta L\right)^{2} \mathrm{e} \\
& -\int_{0}^{z}\left[\mu_{a}\left(\lambda_{\text {emission }}, z_{s}{ }^{\prime}\right)+\mu_{s}\left(\lambda_{\text {emission }}, z_{s}{ }^{\prime}\right)\right] d z_{s}{ }^{\prime}
\end{aligned}
$$

The MCOCT detection sensitivity for each of the coherent emission-based MCOCT methods can be found by substituting the appropriate expression for $C\left(P_{\mathrm{So}}\left(\lambda_{\text {probe }}, z\right)\right)$ into the equation.

The SNR associated with scattering-based MCOCT methods can be analyzed in a similar fashion.

For the engineered scatterer MCOCT method, the detection limit is set by:

$$
\begin{aligned}
& \frac{1}{2}\left(P_{\text {interference,no_contrast_agent }}(\lambda, z)-P_{\text {interference,contrast_agent }}(\lambda, z)\right)(\varepsilon T / \mathrm{h} v) \\
& >\sqrt{\varepsilon P_{\mathrm{Ro}} T / \mathrm{h} v} \sqrt{P_{\mathrm{Ro}} P_{\mathrm{So}}} \mathrm{e}^{-} \int_{0}^{z}\left[\mu_{a}\left(\lambda, z_{s}^{\prime}\right)+\mu_{s}\left(\lambda, z_{s}^{\prime}\right)\right] d z_{s}^{\prime} \\
& \times\left(\frac{1}{2} \frac{n(z) \Delta L \sigma_{\text {backscatter }}}{\sqrt{R_{\text {intrinsic }}(\lambda, z)}}\right)(\varepsilon T / \mathrm{h} v) \\
& >\sqrt{\varepsilon P_{\mathrm{Ro}} T / \mathrm{h} v}
\end{aligned}
$$

where we use the MCOCT signal expression for weak scattering contribution (Eq. 17). The subscript on $P_{\text {interference }}(\lambda, z)$ indicates whether the contrast agent is present or absent. 
The SNR of the method is, therefore, expressible as:

$$
\begin{aligned}
& \mathrm{SNR}_{\mathrm{MCOCT}}=\frac{1}{4}\left(\varepsilon P_{\mathrm{So}} T / \mathrm{h} v\right) \mathrm{e}^{-2 \int_{0}^{z}\left[\mu_{a}\left(\lambda, z_{s}{ }^{\prime}\right)+\mu_{s}\left(\lambda, z_{s}{ }^{\prime}\right)\right] d z_{s}{ }^{\prime}} \\
& \times \frac{\left(n(z) \Delta L \sigma_{\text {backscatter }}(\lambda)\right)^{2}}{R_{\text {intrinsic }}(\lambda, z)} \text {. }
\end{aligned}
$$

The analysis for the magnetically modulated MCOCT is similar, except that the SNR expression will include the additional fractional frequency modulation term $O(\Omega)$.

The following set of equations provides the SNR expressions for the MCOCT methods discussed.

PPOCT:

$$
\begin{aligned}
& \left(\varepsilon P_{\text {So }} T / \mathrm{h} v\right) \times \mid R(z) \mathrm{e}^{-2 \int_{0}^{z_{0}}\left[\mu_{a, \text { intrinsic }}\left(\lambda, z_{s}{ }^{\prime}\right)+\mu_{a, \text { contrast_agent_baseline }}\left(\lambda, z_{s}{ }^{\prime}\right)+\mu_{s}\left(\lambda, z_{s}{ }^{\prime}\right)\right] d z_{s}{ }^{\prime}} \\
& \times \mathrm{e}^{-2 \int_{0}^{z}\left[\mu_{s, \text { reduced }}\left(\lambda_{\text {pump }}, z_{s}{ }^{\prime}\right)+\mu_{a}\left(\lambda_{\text {pump }}, z_{s}{ }^{\prime}\right)\right] d z_{s}{ }^{\prime}} \mid \times\left(I_{\text {pump }, 0} q \tau_{\text {triplet }} \sigma / \mathrm{h} v\right)^{2} \\
& \times(n(z) \Delta \sigma L)^{2}
\end{aligned}
$$

\section{Pump-suppression MCOCT:}

$$
\begin{aligned}
& \left(\varepsilon P_{\mathrm{So}} T \mid \mathrm{h} v\right) \times \mid R(z) \mathrm{e}^{-2 \int_{0}^{z}\left[\mu_{a, \text { intrinsic }}\left(\lambda, z_{s}{ }^{\prime}\right)+\mu_{a, \text { contrast_agent_baseline }}\left(\lambda, z_{s}{ }^{\prime}\right)+\mu_{s}\left(\lambda, z_{s}{ }^{\prime}\right)\right] d z_{s}{ }^{\prime}} \\
& \left.\times \mathrm{e}^{-2 \int_{0}^{z}\left[\mu_{s, \text { reduced }}\left(\lambda_{\text {pump }}, z_{s}{ }^{\prime}\right)+\mu_{a}\left(\lambda_{\text {pump }}, z_{s}{ }^{\prime}\right)\right] d z_{s}{ }^{\prime}}\right) \times\left(I_{\text {pump }, 0} / I_{\text {sat }}\right)^{2}(n(z) \Delta \sigma \Delta L)^{2}
\end{aligned}
$$

\section{Spectral Triangulation MCOCT}

$$
\begin{aligned}
& \left.\left(\varepsilon P_{\mathrm{So}} T \mid \mathrm{h} v\right) \times \mid R(z) \mathrm{e}^{-2 \int_{0}^{z}\left[\mu_{a, \text { intrinsic }}\left(\lambda, z_{s}{ }^{\prime}\right)+\mu_{a, \text { contrast_agent_baseline }}\left(\lambda, z_{s}{ }^{\prime}\right)+\mu_{s}\left(\lambda, z_{s}{ }^{\prime}\right)\right] d z_{s}{ }^{\prime}}\right) \\
& \times(n(z) \Delta \sigma \Delta L / 2)^{2}
\end{aligned}
$$

Second Harmonic OCT:

$$
\begin{aligned}
& (\varepsilon T \mid \mathrm{h} v) \times C_{\mathrm{SHG}} \frac{P_{\mathrm{So}}\left(\lambda_{\text {pump }}, 0\right)^{2}}{A_{\text {pump }}(z)^{2}} B(z)\left(n(z) A\left(\lambda_{\text {emission }}, z\right) \Delta L\right)^{2} \\
& \mathrm{e}^{-\int_{0}^{z_{0}}\left[\mu_{a}\left(\lambda_{\text {emission }}, z_{s}{ }^{\prime}\right)+\mu_{s}\left(\lambda_{\text {emission }}, z_{s}{ }^{\prime}\right)+2 \mu_{a}\left(\lambda_{\text {probe }}, z_{s}{ }^{\prime}\right)+2 \mu_{s}\left(\lambda_{\text {probe }}, z_{s}{ }^{\prime}\right)\right] d z_{s}{ }^{\prime}}
\end{aligned}
$$

\section{NIVI OCT:}

$$
\begin{aligned}
& (\varepsilon T \mid \mathrm{h} v) \times C_{\mathrm{NIVI}}(z) \frac{P_{\mathrm{So}}\left(\lambda_{\text {Stokes }}, 0\right) P_{\mathrm{So}}\left(\lambda_{\mathrm{Pump}}, 0\right)^{2}}{A_{\text {Stokes }}(z) A_{\text {Pump }}(z)^{2}} \\
& \times B(z)\left(n(z) A\left(\lambda_{\text {emission, }}, z\right) \Delta\right)^{2}
\end{aligned}
$$

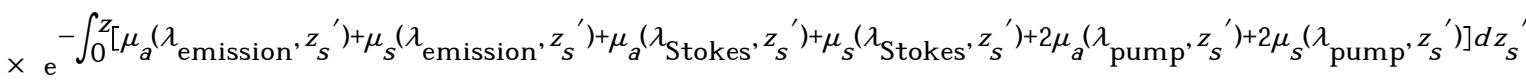


Engineered Scatterer MCOCT:

$$
\begin{aligned}
& \operatorname{SNR}_{\text {MCOCT }}=\frac{1}{4}\left(\varepsilon P_{S o} T / \mathrm{h} v\right) \mathrm{e}^{-2 \int_{0}^{z}\left[\mu_{a}\left(\lambda, z_{s}{ }^{\prime}\right)+\mu_{s}\left(\lambda, z_{s}{ }^{\prime}\right)\right] d z_{s}{ }^{\prime}} \\
& \times \frac{\left(n(z) \Delta L \sigma_{\text {backscatter }}(\lambda)\right)^{2}}{R_{\text {intrinsic }}(\lambda, z)}
\end{aligned}
$$

Magnetically Modulated MCOCT:

$$
\begin{aligned}
& \operatorname{SNR}_{\mathrm{MCOCT}}=\frac{1}{16}\left(\varepsilon P_{S o} T / \mathrm{h} v\right) \times \mathrm{e}^{-2 \int_{0}^{Z}\left[\mu_{a}\left(\lambda, z_{s}{ }^{\prime}\right)+\mu_{s}\left(\lambda, z_{s}{ }^{\prime}\right)\right] d z_{s}{ }^{\prime}} \\
& \times \frac{\left(n(z) \Delta L \sigma_{\text {backscatter }}(\lambda) O(\Omega)\right)^{2}}{R_{\text {intrinsic }}(\lambda, z)}
\end{aligned}
$$

Signal sensitivity in relation to contrast agent concentration-The minimum detectable contrast agent concentration is an extremely important consideration for MCOCT. The relative lack of discussion in the published literature on this aspect hints at the absence of a sufficient theoretical model for addressing this issue; hopefully, the mathematical framework explicitly described in this article will be useful to researchers in this field for tackling this issue. As a rough guide to the acceptable concentration of contrast agent for clinical applications, the FDA-approved dosage of ICG allowed for use in fluorescence angiography is about $10 \mu M$ (103). The rest of this section discusses the relative sensitivity merits of the MCOCT methods in comparison with each other.

One of the more interesting observations that can be made from the SNR expressions associated with the various MCOCT methods is that all of the MCOCT methods exhibit a $n(z)^{2}$ dependency. This dependency has a very important consequence, the relative sensitivity of the MCOCT methods is independent of the contrast agent concentration. In other words, if one MCOCT method is determined to be more sensitive than another MCOCT method for a given contrast agent concentration, we can expect that method to be more sensitive than another at all possible contrast agent concentrations.

This observation may appear to contradict the intuitive notion that coherent emission-based methods should perform better because the amount of coherent emission is proportional to the square of the number of contributing elements within the sample. This intuitive notion is fully valid and can be easily understood by comparing fluorescence microscopy and SHG microscopy. The amount of fluorescence emission generated from a given site is proportional to the concentration of the contrast agent at the given location. In comparison, because of the coherent field generating effect, the amount of second harmonic emission is proportional to the square of the contrast agent's concentration. This implies that there exists a particular contrast agent concentration below which fluorescence microscopy is more sensitive than second harmonic microscopy and above which the situation is reversed. The square dependency in sensitivity with respect to the contrast agent concentration is shared by microscopy methods that have a transmission geometry (such as conventional light microscopy), as well. The underlying mechanism that gives rise to this dependency is very different from that for coherent emission-based methods. In this case, the contrast signal is the difference in the transmitted signal in the presence and absence of the contrast agent. In the limit of low contrast agent concentration, the shot noise term is proportional to the total transmission and is unchanged by presence of the contrast agent. In comparison, the shot noise term is dependent on the contrast agent concentration for fluorescence microscopy. This insensitivity of the noise term to contrast agent concentration leads to a contrast agent 
concentration squared dependency for transmission microscopy. Similar to second harmonic microscopy, it is less sensitive than fluorescence microscopy under a certain contrast agent concentration threshold.

The same sensitivity analysis arguments can be applied to the various MCOCT methods. In the shot noise limit, we can expect the various absorption-based or scattering-based methods to have the same theoretical SNR as transmission microscopy experiments with the same parameters, and the coherent emission-based methods to have the same theoretical SNR as second harmonic or CARS microscopy experiments with the same parameters.

We should also note the differences in the relative sensitivity of the methods with respect to the absorption, scattering and conversion efficiency effective cross-sections of the various methods. The terms $\Delta \sigma$ (from Eqs. $7 \mathrm{~b}$ and 12), $\sigma_{\text {backscatter }}(\lambda)$ (from Eqs. 16 and 17), $C_{\mathrm{SHG}}\left(P_{\mathrm{So}}\left(\lambda_{\text {pump }}, 0\right) / A_{\text {pump }}(z)^{2}\right)$ (from Eq. 14) and $C_{\mathrm{NIVI}}(z) P_{\mathrm{So}}\left(\lambda_{\mathrm{Stokes}}, 0\right) P_{\mathrm{So}}\left(\lambda_{\text {pump }}, 0\right)$ (from Eq. 15), all represent a cross-sectional efficiency of the contrast agent used in the specific method for returning an optical signal that is amendable to interferometry detection. Given the relative inefficiency of the nonlinear methods in comparison with simple absorption, we can expect $C_{\mathrm{SHG}}\left(P_{\mathrm{So}}\left(\lambda_{\text {pump }}, 0\right) / A_{\text {pump }}(z)^{2}\right)$ and $C_{\mathrm{NIVI}}(z) P_{\mathrm{So}}\left(\lambda_{\mathrm{Stokes}}, 0\right)-P_{\mathrm{So}}\left(\lambda_{\text {pump }}, 0\right)$ to be smaller than $\Delta \sigma$ in a typical situation. Because the scatterer-based methods rely on the use of micron size scattering objects, $\sigma_{\text {backscatter }}(\lambda)$ can be expected to be orders of magnitude bigger than $\Delta \sigma$. Therefore, approximately speaking, given the same contrast agent number concentration, we can expect the scatterer-based MCOCT methods to be more sensitive than the absorptionbased methods. The absorption-based methods are, in turn, likely to be more sensitive than the coherent emission-based methods.

Signal sensitivity in terms of depth attenuation-The above SNR equations can also permit us to gauge the relative performance of the MCOCT methods in terms of their depth imaging potential. In general, we can expect the scattering of the biological target to be the major attenuation contributor in the OCT imaging process. The reduced scattering extinction coefficients and the intrinsic absorption extinction coefficients are generally an order of magnitude lower than the scattering extinction coefficients. Therefore, we may ignore the attenuation contribution of the reduced scattering and intrinsic absorption extinction coefficients in our discussion of the depth penetration capability of the various MCOCT methods.

The class of absorption-based and scattering-based MCOCT methods can be expected to perform favorably in terms of depth penetration because SNR attenuation is dependent on only twice the product of the scattering extinction coefficient and the depth of interest (the factor of two is due to the double pass through the sample). This dependency is no worse than that for a conventional OCT imaging system. In other words, the absorption-based MCOCT methods do not carry any additional attenuation-based SNR overhead that is not already present in a conventional OCT imaging system.

The coherent emission-based MCOCT methods can be expected to have shallower depth penetration. In the case of SH-OCT, the SNR attenuation is proportional to three times the scattering extinction coefficients (two at the pump wavelength and one at the emission wavelength). The emission wavelength scattering extinction coefficient is particularly troublesome because the second harmonic emission is at a shorter wavelength. Therefore, the scattering attenuation for the emitted light can be significantly larger than that for the pump light. In the case of NIVI-based MCOCT, the SNR attenuation is dependent on four times the scattering extinction coefficients. The high degree of dependency is mitigated to some extent by the fact that it is, in principle, possible to arrange all the wavelengths involved to fall within a narrow range so that all the scattering extinction coefficients are minimized to some extent. 
Speckle artifact limitation-PPOCT and pump-suppression MCOCT share a common obstacle that can potentially prevent them from achieving the SNR limit discussed above. The issue is that temporal speckle artifacts pervade OCT imaging of biological tissue $(104,105)$. The temporal speckle phenomenon can be attributed to two effects: (1) the relative movement of scatterers within the coherence volume, as defined by the lateral focal spot size and the axial coherence length, and (2) the movement of scatterers into and out of the coherence volume and the illumination volume above the targeted coherence volume. The first effect creates temporal speckles because light scattered from scattering sites that are close to each other can constructively or destructively interfere, resulting in either a larger or smaller effective reflectivity $R\left(\lambda, x_{s}\right)$. Any relative movement of the scatterers will alter the interference and create a net increase or decrease in the amount of light that is backscattered to the OCT detection system. This effect is very pronounced when the number of scattering sites within the coherence volume is small ( 2-100) and is diminished when the number is very small or very large. When the scattering sites are far and few, it will be unlikely for any given coherence volume to have more than one scattering site, and the backscattered light will not have cross interference terms. In the limit where the scattering site density is high and the sites are randomly oriented, the resulting reflectivity will be approximately the sum of the individual reflectivity; interference effect effectively averages out. The second effect, movement of scatterers into and out of the coherence volume, can also cause a fluctuation in $R\left(\lambda, x_{s}\right)$. In addition, it can also change the effective $\mu_{s}\left(\lambda, x_{s}\right)$ in Eq. (2) because the movement of scattering sites away from the illumination volume above the targeted coherence volume will effectively increase the amount of ballistically propagating light that is detected by the OCT system.

Because pump-probe MCOCT and pump-suppression MCOCT extract molecular contrast information by comparing and taking the difference of two sequential OCT scans, any significant time difference between the acquisitions of the two scans will result in changes in $R\left(\lambda, x_{s}\right)$ and $\mu_{s}\left(\lambda, x_{s}\right)$. The changes can alter the OCT scan signal to the extent that the contrastinduced signal difference is masked. Speckle artifacts have always been an issue in the context of OCT imaging because they can manifest as image graininess that varies with time. In a recent publication, a research group reported a speckle decorrelation time constant for aortic tissue and aortic plaques that varies from 40 to $500 \mathrm{~ms}$ (45). To minimize the effect of speckle artifacts on the contrast signal, the sequential OCT scans acquired in PPOCT and pumpsuppression MCOCT must occur within a time window that is much less than the speckle decorrelation time.

The implementation of PPOCT as reported in Ref. (62) is able to satisfy this condition well, the time separation between scan pairs are $0.5 \mathrm{~ms}$. However, the implementation of pumpsuppression MCOCT as reported in Ref. (63) is unable to satisfy the condition, the resulting speckle artifact-induced errors have to be minimized by large sample averaging. Future implementations of these two MCOCT methods should take the temporal speckle artifact issues into due consideration during system design.

The scattering-based MCOCT methods' SNR may be diminished to a certain extent by speckle artifacts because the contrast agent concentration to signal strength relationship given by Eq. (22) is an approximation in which speckle artifacts are ignored. One solution, albeit a highly inefficient one in terms of time investment, is to allow for a sufficiently long averaging time base for signal collection.

\section{CONCLUSIONS}

By virtue of the wide range of possible light-matter interaction mechanisms, there are many approaches for mapping contrast agents in an OCT imaging modality. In this review, we have highlighted the various implementation approaches that have been reported thus far. They can 
be approximately categorized as absorption-based MCOCT methods, coherent emission-based MCOCT methods and scattering-based MCOCT methods.

One of the absorption-based methods, pump-suppression MCOCT, is particularly promising as a method for mapping endogenously expressed contrast agents because it is well suited for mapping photoswitchable proteins such as bR and phyA. Two other absorption-based methods, spectroscopic OCT and spectral triangulation MCOCT, have demonstrated the ability to observe an FDA-approved dye, ICG, in plant and animal models. A coherent emission-based method, NIVI, may potentially be used to image a very wide range of chemical or biochemical contrast agents by locking to their CARS emission spectral profiles. The other coherent emission-based method, SHG OCT, may be used to not only map a contrast agent's distribution but also its structural orientation. The two scattering-based MCOCT methods, which use macroscopic particles engineered to strongly scatter light, provide yet another set of MCOCT methods that are worth investigation and research.

Although the technical development of MCOCT methods is progressing at a healthy pace, the field is presently lacking in research efforts into the search or engineering (or both) of appropriate contrast agents and the development of methods for functionalizing the contrast agents to appropriately bind to biochemical species of interest. These important aspects of MCOCT research deserve the attention of chemical engineers and biochemistry researchers. Some of the pertinent questions in the field are: (1) Is it possible to engineer and adapt some of the existing genetically expressible fluorophore, such as GFP, to serve as contrast agents for MCOCT imaging? (2) How do we engineer some of the contrast agents to optimize on their contrast rendering capability? (3) How do we functionalize the contrast agents that have been demonstrated to work well in MCOCT methods so that they can bind to a biochemical species of interest?

MCOCT imaging methods can potentially have wide and very significant effect on the way clinicians diagnose diseases and the conduct of biomedical research.

\section{Acknowledgements}

The author gratefully acknowledges useful comments from Brian Applegate (Duke University), Stephen Boppart (University of Illinois at Urbana-Champaign), Zhongping Chen (UC Irvine), Eric Potma (Harvard University), Zahid Yaqoob (Caltech), Xin Heng (Caltech), Fei Wang (Caltech). The author also thanks the various authors of the original MCOCT papers for permission to use their figures in this review.

\section{References}

1. Valk, P. E., D. L. Bailey, D. W. Townsend and M. N. Maisey (2003) Positron Emission Tomography: Principles and Practice. Springer Verlag, New York.

2. Fernandes PA, Carvalho ATP, Marques AT, Pereira ALF, Madeira APS, Ribeiro ASP, Carvalho AFR, Ricardo ETA, Pinto FJV, Santos HA, Mangericao HDG, Martins HM, Pinto HDB, Santos HRR, Moreira S, Azeredo MJV, Abreu RPS, Oliveira RMS, Sousa SFM, Silva R, Mourao ZS, Ramos MJ. New designs for MRI contrast agents. J Comput Aided Mol Des 2003;17:463-473. [PubMed: 14677640]

3. Denk W, Strickler JH, Webb WW. 2-photon laser scanning fluorescence microscopy. Science 1990;248:73-76. [PubMed: 2321027]

4. Drezek R, Brookner C, Pavlova I, Boiko I, Malpica A, Lotan R, Follen M, Richards-Kortum R. Autofluorescence microscopy of fresh cervical-tissue sections reveals alterations in tissue biochemistry with dysplasia. Photochem Photobiol 2001;73:636-641. [PubMed: 11421069]

5. Heldal M, Norland S, Bratbak G, Riemann B. Determination of bacterial-cell number and cell-volume by means of flow-cytometry, transmission electron-microscopy, and epifluorescence microscopy. J Microbiol Methods 1994;20:255-263. 
6. Qu JY, Hua JW. Calibrated fluorescence imaging of tissue in vivo. Appl Phys Lett 2001;78:40404042.

7. Wagnieres GA, Star WM, Wilson BC. In vivo fluorescence spectroscopy and imaging for oncological applications. Photochem Photobiol 1998;68:603-632. [PubMed: 9825692]

8. Duggar KH, Lauffenburger DA, So PTC. Two-photon fluorescence resonance energy transfer and its application to measurement of cell adhesion receptor binding to substratum ligands. Biophys $\mathbf{J}$ 1999;76:A98-A98.

9. Hoppe A, Christensen K, Swanson JA. Fluorescence resonance energy transfer-based stoichiometry in living cells. Biophys J 2002;83:3652-3664. [PubMed: 12496132]

10. Malvezzi-Campeggi F, Jahnz M, Heinze KG, Dittrich P, Schwille P. Light-induced flickering of DsRed provides evidence for distinct and interconvertible fluorescent states. Biophys J 2001;81:1776-1785. [PubMed: 11509387]

11. Srivastava M, Ow H, Larson D, Holowka D, Wiesner U, Webb W, Baird B. Single particle tracking of fluorescent silica nanoparticles bound to IgE receptors on RBL mast cells. Biophys J 2002;82:2436. [PubMed: 11964232]

12. Loerke D, Preitz B, Stuhmer W, Oheim M. Super-resolution measurements with evanescent-wave fluorescence excitation using variable beam incidence. J Biomed Opt 2000;5:23-30. [PubMed: 10938762]

13. Oheim M, Loerke D, Chow RH, Stuhmer W. Evanescent-wave microscopy: a new tool to gain insight into the control of transmitter release. Philos Trans R Soc Lond B Biol Sci 1999;354:307-318. [PubMed: 10212479]

14. Panchuk-Voloshina N, Haugland RP, Bishop-Stewart J, Bhalgat MK, Millard PJ, Mao F, Leung WY. Alexa dyes, a series of new fluorescent dyes that yield exceptionally bright, photostable conjugates. J Histochem Cytochem 1999;47:1179-1188. [PubMed: 10449539]

15. Gerdes HH, Kaether C. Green fluorescent protein: applications in cell biology. FEBS Lett 1996;389:44-47. [PubMed: 8682203]

16. Heikal AA, Hess ST, Webb WW. Multiphoton molecular spectroscopy and excited-state dynamics of enhanced green fluorescent protein (EGFP): acid-base specificity. Chem Phys 2001;274:37-55.

17. Jung G, Mais S, Zumbusch A, Brauchle C. The role of dark states in the photodynamics of the green fluorescent protein examined with two-color fluorescence excitation spectroscopy. J Phys Chem A 2000;104:873-877.

18. Kohler RH, Zipfel WR, Webb WW, Hanson MR. The green fluorescent protein as a marker to visualize plant mitochondria in vivo. Plant J 1997;11:613-621. [PubMed: 9107047]

19. Heikal AA, Hess ST, Baird GS, Tsien RY, Webb WW. Molecular spectroscopy and dynamics of intrinsically fluorescent proteins: coral red (dsRed) and yellow (Citrine). Proc Natl Acad Sci USA 2000;97:11996-12001. [PubMed: 11050231]

20. Gratz H, Penzkofer A, Abels C, Szeimies RM, Landthaler M, Baumler W. Photo-isomerisation, triplet formation, and photo-degradation dynamics of indocyanine green solutions. J Photochem Photobiol A: Chem 1999;128:101-109.

21. Yun S, Tearney G, Bouma B, Park B, de Boer JF. High-speed spectral domain optical coherence tomography at $1.3 \mu \mathrm{m}$ wavelength. Opt Express 2003;11:3598-3604.

22. Levine R, Brucker AJ, Robinson F. Long-term follow-up of idiopathic central serous chorioretinography by fluorescein angiography. Ophthalmology 1989;96(6):854-859. [PubMed: 2740080]

23. Buschman HP, Deinum G, Motz JT, Fitzmaurice M, Kramer JR, van der Laarse A, Bruschke AV, Feld MS. Raman microspectroscopy of human coronary atherosclerosis: biochemical assessment of cellular and extracellular morphologic structures in situ. Cardiovasc Pathol 2001;10:69-82. [PubMed: 11425600]

24. Buschman HP, Motz JT, Deinum G, Romer TJ, Fitzmaurice M, Kramer JR, van der Laarse A, Bruschke AV, Feld MS. Diagnosis of human coronary atherosclerosis by morphology-based Raman spectroscopy. Cardiovasc Pathol 2001;10:59-68. [PubMed: 11425599]

25. Freund I, Deutsch M, Sprecher A. Connective-tissue polarity-optical 2nd-harmonic microscopy, crossed-beam summation, and small-angle scattering in rat-tail tendon. Biophys J 1986;50:693-712. [PubMed: 3779007] 
26. Georgiou E, Theodossiou T, Hovhannisyan V, Politopoulos K, Rapti GS, Yova D. Second and third optical harmonic generation in type I collagen, by nanosecond laser irradiation, over a broad spectral region. Opt Commun 2000;176:253-260.

27. Guo YC, Ho PP, Savage H, Harris D, Sacks P, Schantz S, Liu F, Zhadin N, Alfano RR. Secondharmonic tomography of tissues. Opt Lett 1997;22:1323-1325.

28. Cheng JX, Volkmer A, Book LD, Xie XS. An epi-detected coherent anti-stokes raman scattering (ECARS) microscope with high spectral resolution and high sensitivity. J Phys Chem B 2001;105:1277-1280.

29. Volkmer A, Cheng JX, Book LD, Xie XS. New advances in coherent anti-Stokes Raman scattering (CARS) microscopy and spectroscopy of biological systems. Biophys J 2001;80:164A-164A.

30. Zumbusch A, Holtom GR, Xie XS. Three-dimensional vibrational imaging by coherent anti-Stokes Raman scattering. Phys Rev Lett 1999;82:4142-4145.

31. Hee MR, Swanson EA, Huang D, Izatt JA, Lin CP, Schuman JS, Puliafito CA, Fujimoto JG. Optical coherence tomography. Invest Ophthalmol Vis Sci 1992;33:722-722.

32. Tearney GJ, Bouma BE, Boppart SA, Golubovic B, Swanson EA, Fujimoto JG. Rapid acquisition of in vivo biological images by use of optical coherence tomography. Opt Lett 1996;21:1408-1410.

33. Fercher AF, Hitzenberger CK, Sticker M, Moreno-Barriuso E, Leitgeb R, Drexler W, Sattmann H. A thermal light source technique for optical coherence tomography. Opt Commun 2000;185:57-64.

34. Rollins AM, Kulkarni MD, Yazdanfar S, Ung-arunyawee R, Izatt JA. In vivo video rate optical coherence tomography. Opt Express 1998;3:219.

35. Schmitt JM. Optical coherence tomography (OCT): a review. IEEE J Selected Top Quantum Electron 1999;5:1205-1215.

36. Bouma, B. and G. Tearney (2001) Handbook of Optical Coherence Tomography. Marcel Dekker, New York, New York.

37. American National Standards Institute (2000) Safe use of lasers. In ANSI Z 136, pp. 1-2000. Laser Institute of America, Orlando, FL.

38. Yang CH, Wax A, Feld MS. Measurement of the anomalous phase velocity of ballistic light in a random medium by use of a novel interferometer. Opt Lett 2001;26:235-237. [PubMed: 18033558]

39. Hee MR, Izatt JA, Swanson EA, Huang D, Schuman JS, Lin CP, Puliafito CA, Fujimoto JG. Optical coherence tomography of the human Retina. Arch Ophthalmol 1995;113:325-332. [PubMed: 7887846]

40. Yazdanfar S, Rollins AM, Izatt JA. Imaging and velocimetry of the human retinal circulation with color Doppler optical coherence tomography. Opt Lett 2000;25:1448-1450.

41. Huang D, Chalita MR, Li Y, Lowder CY, Meisler DM, Rollins AM, Izatt JA. High-speed optical coherence tomography of anterior segment surgical anatomy and pathology. Invest Ophthalmol Vis Sci 2003;44:U141-U141.

42. Li Y, Chalita MR, Goldsmith J, Westphal V, Bower BA, Shekhar R, Rollins AM, Izatt JA, Huang D. Automated anterior chamber biometry with high-speed optical coherence tomography. Invest Ophthalmol Vis Sci 2003;44:U285-U285.

43. Radhakrishnan S, Rollins AM, Roth JE, Yazdanfar S, Westphal V, Bardenstein DS, Izatt JA. Realtime optical coherence tomography of the anterior segment at $1310 \mathrm{~nm}$. Arch Ophthalmol 2001;119:1179-1185. [PubMed: 11483086]

44. Brezinski ME, Tearney GJ, Bouma BE, Izatt JA, Hee MR, Swanson EA, Southern JF, Fujimoto JG. Optical coherence tomography for optical biopsy-properties and demonstration of vascular pathology. Circulation 1996;93:1206-1213. [PubMed: 8653843]

45. Tearney G, Bouma B. Atherosclerotic plaque characterization by spatial and temporal speckle pattern analysis. Opt Lett 2002;27:533-535. [PubMed: 18007856]

46. Das A, Sivak MV, Chak A, Wong RC, Westphal V, Rollins AM, Izatt J, Isenberg GA, Willis J. Role of high resolution endoscopic imaging using optical coherence tomography (OCT) in patients with Barrett's esophagus (BE). Gastrointest. Endosc 2000;51:AB93-AB93.

47. Sivak MV, Kobayashi K, Izatt JA, Rollins AM, Ung-runyawee R, Chak A, Wong RCK, Isenberg GA, Willis J. High-resolution endoscopic imaging of the GI tract using optical coherence tomography. Gastrointest Endosc 2000;51:474-479. [PubMed: 10744825] 
48. Rollins AM, Ung-arunyawee R, Chak A, Wong RCK, Kobayashi K, Sivak MV, Izatt JA. Real-time in vivo imaging of human gastrointestinal ultrastructure by use of endoscopic optical coherence tomography with a novel efficient interferometer design. Opt Lett 1999;24:1358-1360.

49. Sokolov K, Follen M, Aaron J, Pavlova I, Malpica A, Lotan R, Richards-Kortum R. Real-time vital optical imaging of precancer using anti-epidermal growth factor receptor antibodies conjugated to gold nanoparticles. Cancer Res 2003;63:1999-2004. [PubMed: 12727808]

50. Swanson E, Huang D, Hee M, Fujimoto JG, Lin CP, Puliafito CA. High-speed optical coherence domain reflectometry. Opt Lett 1992;17:151-153.

51. Cheong W, Prahl SA, Welch AJ. A review of the optical properties of biological tissue. IEEE J Selected Top Quantum Electron 1990;26:2166-2185.

52. Kienle A, Lilge L, Patterson MS, Hibst R, Steiner R, Wilson BC. Spatially resolved absolute diffuse reflectance measurements for noninvasive determination of the optical scattering and absorption coefficients of biological tissue. Appl Opt 1996;35:2304-2314.

53. Fercher AF, Hitzenberger CK, Kamp G, Elzaiat SY. Measurement of intraocular distances by backscattering spectral interferometry. Opt Commun 1995;117:43-48.

54. Hausler G, Lindner MW. "Coherence Radar" and "Spectral Radar"-new tools for dermatological diagnosis”. J Biomed Opt 1998;3:21.

55. White B, Pierce M, Nassif N, Cense B, Park B, Tearney G, Bouma B, Chen T, de Boer JF. In vivo dynamic human retinal blood flow imaging using ultra-high-speed spectral domain optical Doppler tomography. Opt Express 2003;11:3490-3497.

56. Choma MA, Sarunic M, Yang C, Izatt JA. Sensitivity advantage of swept source and Fourier domain optical coherence tomography. Opt Express 2003;11:2183-2189.

57. de Boer JF, Cense B, Park BH, Pierce MC, Teamey GJ, Bouma BE. Improved signal-to-noise ratio in spectral-domain compared with time-domain optical coherence tomography. Opt Lett 2003;28:2067-2069. [PubMed: 14587817]

58. Leitgeb R, Hitzenberger CK, Fercher AF. Performance of fourier domain vs. time domain optical coherence tomography. Opt Express 2003;11:889-894.

59. Yun SH, Boudoux C, Tearney GJ, Bouma BE. High-speed wavelength-swept semiconductor laser with a polygon-scanner-based wavelength filter. Opt Lett 2003;28:1981. [PubMed: 14587796]

60. Povazay B, Bizheva K, Unterhuber AH, Hermann B, Sattmann H, Fercher AF, Drexler W, Apolonski A, Wadsworth WJ, Knight JC, Russell PSJ, Vetterlein M, Scherzer E. Submicrometer axial resolution optical coherence tomography. Opt Lett 2002;27:1800. [PubMed: 18033368]

61. Wojtkowski M, Srinivasan VJ, Ko T, Fujimoto JG, Kowalczyk A, Duker JS. Ultrahigh-resolution, high-speed, Fourier domain optical coherence tomography and methods for dispersion compensation. Opt Express 2004;12:2404.

62. Rao KD, Choma MA, Yazdanfar S, Rollins AM, Izatt JA. Molecular contrast in optical coherence tomography by use of a pump-probe technique. Opt Lett 2003;28:340-342. [PubMed: 12659437]

63. Yang C, Choma MA, Lamb LE, Simon JD, Izatt J. Protein based molecular contrast OCT with phytochrome as the contrast agent. Opt Lett 2004;29:1396. [PubMed: 15233447]

64. Xu C, Ye J, Marks DL, Boppart SA. Near-infrared dyes as contrast-enhancing agents for spectroscopic optical coherence tomography. Opt Lett 2004;29:1647. [PubMed: 15309847]

65. Ye, J. and S. A. Boppart (2003) Spectroscopic OCT of infrared dyes as contrast agents. In CLEO/ QELS. 2003. Baltimore, MD.

66. Yang C, Lamb LE, Simon JD, Choma MA, Applegate B, Izatt J. Spectral triangulation molecular contrast optical coherence tomography with indocyanine green as the contrast agent. Opt Lett 2004;29:2016-2018. [PubMed: 15455765]

67. Clark, G. and H. J. Conn (1981) Staining Procedures. Williams and Wilkins, Baltimore, MD.

68. Canto MI. Methylene blue chromoendoscopy for Barrett's esophagus: Coming soon to your GI unit. Gastrointest Endosc 2001;54:403. [PubMed: 11522995]

69. Haupst U, Tittor J, Oesterhelt D. Closing in on bacteriorhodopsin: progress in understanding the molecule. Annu Rev Biophys Biomol Struct 1999;28:367. [PubMed: 10410806]

70. Fankhauser C. The phytochromes, a family of red/far-red absorbing photoreceptors. J Biol Chem 2001;276:11453-11456. [PubMed: 11279228] 
71. Gartner W, Hill C, Worm K, Braslavsky SE, Schaffner K. Influence of expression system on chromophore binding and preservation of spectral properties in recombinant phytochrome A. Eur. J Biochem 1996;236:978-983.

72. Kidd DG, Lagarias JC. Phytochrome from the Green Alga Mesotaenium caldariorum. J Biol Chem 1990;265:7029-7035. [PubMed: 2324111]

73. Morgner U, Drexler W, Kartner FX, Li XD, Pitris C, Ippen EP, Fujimoto JG. Spectroscopic optical coherence tomography. Opt Lett 2000;25:111-113. [PubMed: 18059799]

74. Jiang Y, Tomov I, Wang Y, Chen ZP. Second-harmonic optical coherence tomography. Opt Lett 2004;29:1090. [PubMed: 15181995]

75. Yazdanfar S, Laiho LH, So PTC. Interferometric second harmonic generation microscopy. Opt Express 2004;12:2739.

76. Applegate B, Yang C, Rollins AM, Izatt J. Polarization resolved second harmonic generation optical coherence tomography in collagen. Opt Lett 2004;29:2252. [PubMed: 15524371]

77. Vinegoni C, Bredfeldt JS, Marks DL, Boppart SA. Nonlinear optical contrast enhancement for optical coherence tomography. Opt Express 2004;12:331-341.

78. Hendrickx E, Clays K, Persoons A, Dehu C, Bredas JL. The bacteriorhodopsin chromophore retinal and derivatives: An experimental and theoretical investigation of the 2nd-order optical properties. $J$ Am Chem Soc 1995;117:3547-3555.

79. Song Q, Wan CZ, Johnson CK. Time-resolved 2nd-harmonic generation in the randomly oriented purple membrane. J Phys Chem 1994;98:1999-2001.

80. Izatt JA, Hee MR, Owen GM, Swanson EA, Fujimoto JG. Optical coherence microscopy in scattering media. Opt Lett 1994;19:590-592.

81. Butcher, P. N. and D. Cotter (1990) The elements of nonlinear optics. In Cambridge Studies in Modern Optics, Vol. 9 (Edited by) Cambridge University Press, Cambridge.

82. Marks DL, Boppart SA. Nonlinear interferometric vibrational imaging. Phys. Rev. Lett 2004;92:123905-1-123905-4. [PubMed: 15089675]

83. Drexler W, Morgner U, Kartner FX, Pitris C, Boppart SA, Li XD, Ippen EP, Fujimoto JG. In vivo ultrahigh-resolution optical coherence tomography. Opt Lett 1999;24:1221-1223.

84. Marks DL, Oldenburg AL, Reynolds JJ, Boppart SA. Study of an ultrahigh-numerical-aperture fiber continuum generation source for optical coherence tomography. Opt Lett 2002;27:2010-2012. [PubMed: 18033428]

85. Lee TM, Oldenburg AL, Sitafalwalla S, Marks DL, Luo W, Toublan FJ, Suslick KS, Boppart SA. Engineered microsphere contrast agents for optical coherence tomography. Opt Lett 2003;28:15461548. [PubMed: 12956374]

86. Oldenburg, A. L., J. R. Gunther, D. L. Marks, F. J. Toublan, K. S. Suslick and S. A. Boppart (2003) Selective OCT imaging of cells using magnetically modulated optical contrast agents. In CLEO/ QELS conference in Baltimore, Maryland.

87. Holtom GR, Thrall BD, Chin BY, Wiley HS, Colson SD. Achieving molecular selectivity in imaging using multiphoton Raman spectroscopy techniques. Traffic 2001;2:781-788. [PubMed: 11733044]

88. Arnold J, Bouche T, Dreier T, Wichmann J, Wolfrum J. Cars studies on the heterogenous relaxation of vibrationally excited hydrogen and deuterium. Chem Phys Lett 1993;203:283-288.

89. Hill JR, Moore DS, Schmidt SC, Storm CB. Infrared, Raman, and coherent anti-Stokes-Raman Spectroscopy of the hydrogen-deuterium isotopomers of nitromethane. J Phys Chem 1991;95:30373044.

90. Creemers TMH, Lock AJ, Subramaniam V, Jovin TM, Volker S. Red-shifted mutants of green fluorescent protein: reversible photoconversions studied by hole-burning and high-resolution spectroscopy. Chem Phys 2002;275:109-121.

91. Garcia-Parajo MF, Segers-Nolten GMJ, Veerman JA, Greve J, van Hulst NF. Real-time light-driven dynamics of the fluorescence emission in single green fluorescent protein molecules. Proc Natl Acad Sci USA 2000;97:7237-7242. [PubMed: 10860989]

92. Widengren J, Mets U, Rigler R. Photodynamic properties of green fluorescent proteins investigated by fluorescence correlation spectroscopy. Chem Phys 1999;250:171-186.

93. Bouas-Laurent H, Durr H. Organic photochromism. Pure Appl Chem 2001;73:639-665. 
94. Crano J, Knowles D, Kwiatkowski P, Flood T, Ross R, Chiang L, Lasch J, Chadha R, Siuzdak G. Structure of 3 novel photochromic compounds-X-ray crystallographic and theoretical-studies. Acta Crystallogr B 1994;50:772-779.

95. Smets G, Braeken J, Irie M. Photomechanical effects in photochromic systems. Pure Appl Chem 1978;50:845-856.

96. Bechem M, Beutner S, Burkhardt N, Fuchs C, Kryschi C, Paffhausen W, Reiffers B, Schade A, Schlue WR, Schmid D, Schneider L, Schulte P, Wimmer T, Witzak D, Martin HD. Novel hyperpolarizable and fluorescent dyes in lipid membranes: studying membrane potentials using nonlinear optical and fluorescence. Electrochim Acta 2003;48:3387-3393.

97. Moreaux L, Sandre O, Mertz J. Membrane imaging by second-harmonic generation microscopy. J Opt Soc Am B Opt Phys 2000;17:1685-1694.

98. Moreaux L, Sandre O, Blanchard-Desce M, Mertz J. Membrane imaging by simultaneous secondharmonic generation and two-photon microscopy. Opt Lett 2000;25:320-322. [PubMed: 18059867]

99. Bouevitch O, Lewis A, Pinevsky I, Wuskell JP, Loew LM. Probing membrane-potential with nonlinear optics. Biophys J 1993;65:672-679. [PubMed: 8218895]

100. Schmidt R, Bodesheim M. Efficiencies of O2((1)Sigma(G)(+)) and O2((1)Delta(G)) formation in the primary steps of triplet-state photosensitization in solution. Chem Phys Lett 1993;213:111-116.

101. Konig K, Becker TW, Fischer P, Riemann I, Halbhuber KJ. Pulse-length dependence of cellular response to intense near- infrared laser pulses in multiphoton microscopes. Opt Lett 1999;24:113115.

102. Konig K, So PTC, Mantulin WW, Gratton E. Cellular response to near-infrared femtosecond laser pulses in two-photon microscopes. Opt Lett 1997;22:135-136.

103. Stanga PE, Lim JI, Hamilton P. Indocyanine green angiography in choriorefinal diseases: indications and interpretation—an evidence-based update. Ophthalmology 2003;110:15-21. [PubMed: 12511340]

104. Bashkansky M, Reintjes J. Statistics and reduction of speckle in optical coherence tomography. Opt Lett 2000;25:545-547. [PubMed: 18064106]

105. Schmitt JM, Xiang SH, Yung KM. Speckle in optical coherence tomography. J Biomed Opt 1999;4:95-105.

\section{APPENDIX 1}

This appendix derives the number concentration of molecules that are shelved into the triplet state in the steady state situation. We assume that a pump beam of intensity $I_{\text {pump }}$ and of photon energy quanta, $\mathrm{h} v_{g-s}$, matches with the resonant transition energy between the first excited singlet and ground state is incident on the molecules. The associated transition rates equations are:

$$
\begin{gathered}
\frac{d n_{\text {singlet }}}{d t}=\left(\frac{I_{\text {pump }} \sigma_{g-s}}{\mathrm{~h} v_{g-s}}\right)\left(n_{\text {ground }}-n_{\text {singlet }}\right)-\frac{n_{\text {singlet }}}{\tau_{\text {singlet }}}-\frac{n_{\text {singlet }}}{\tau_{\text {ISC }}} \\
\frac{d n_{\text {ground }}}{d t}=-\left(\frac{I_{\text {pump }} \sigma_{g-s}}{\mathrm{~h} v_{g-s}}\right)\left(n_{\text {ground }}-n_{\text {singlet }}\right)+\frac{n_{\text {singlet }}}{\tau_{\text {singlet }}}+\frac{n_{\text {triplet }}}{\tau_{\text {triplet }}} \\
\frac{d n_{\text {triplet }}}{d t}=\frac{n_{\text {singlet }}}{\tau_{\text {ISC }}}-\frac{n_{\text {triplet }}}{\tau_{\text {triplet }}}
\end{gathered}
$$

where $n_{\text {ground }}, n_{\text {singlet }}$ and $n_{\text {triplet }}$ are the number concentrations of molecules in the ground state, singlet state and triplet state, respectively. $\tau_{\text {singlet }}, \tau_{\text {ISC }}$ and $\tau_{\text {triplet }}$ are the decay time constants associated with the singlet to ground state transition, the singlet to triplet state transition and the triplet to ground state transition, respectively. $\sigma_{g-s}$ is the absorption crosssection associated with the ground to singlet state transition. 
To simplify the equations, we shall use the following three quantities:

Total number concentration of the molecules:

$$
n_{o}=n_{\text {ground }}+n_{\text {singlet }}+n_{\text {triplet }}
$$

Fluorescence quantum yield:

$$
q=\tau_{\mathrm{ISC}} / \tau_{\mathrm{ISC}}+\tau_{\text {singlet }}
$$

Saturation intensity:

$$
I_{\text {sat }}=\mathrm{h} v_{g-s} / \sigma_{g-s} \tau_{\text {singlet }}
$$

Finally, because the transition to the triplet state is a rare event and the triplet state lifetime is much longer than the other lifetimes involved, we shall assume that $(1-q) \ll 1$ and $\tau_{\text {triplet }} \gg$ $\tau_{\text {singlet }}$.

By making these assumptions and setting the three rate equations to zero in accordance with the equilibrium condition, we obtain:

$$
\begin{gathered}
n_{\text {singlet }} \approx n_{o} \frac{1}{2+\left(I_{\text {sat }} / I_{\text {pump }}\right)} \\
n_{\text {triplet }}=\frac{\tau_{\text {triplet }}}{\tau_{\text {ISC }}} n_{\text {singlet }} \approx(1-q) n_{\text {singlet }}
\end{gathered}
$$

The combination of the two equations gives:

$$
n_{\text {triplet }} \approx(1-q) n_{o} \frac{1}{2+\left(I_{\text {sat }} / I_{\text {pump }}\right)} \approx(1-q) n_{o} \frac{I_{\text {Pump }}}{I_{\text {Sat }}} \text {, }
$$

where the last approximation is derived in the situation in which $I_{\text {pump }}$ is much smaller than $I_{\text {sat. }}$. 


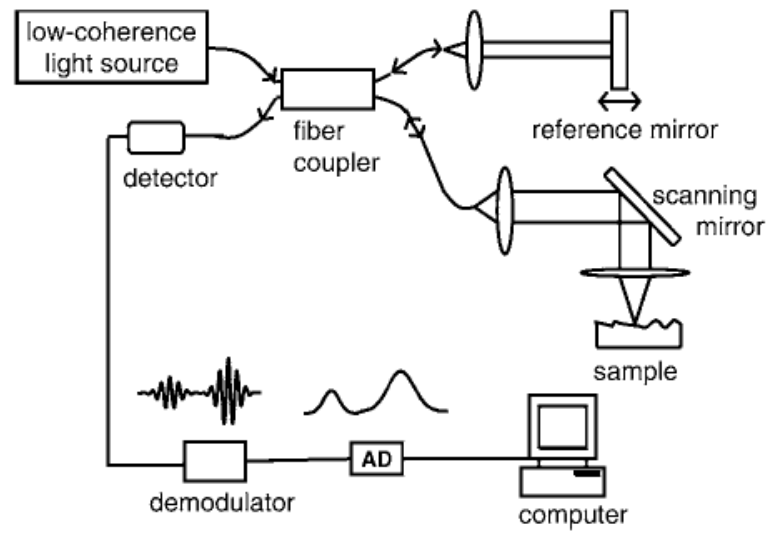

Figure 1.

Experimental scheme of a time domain OCT system. 


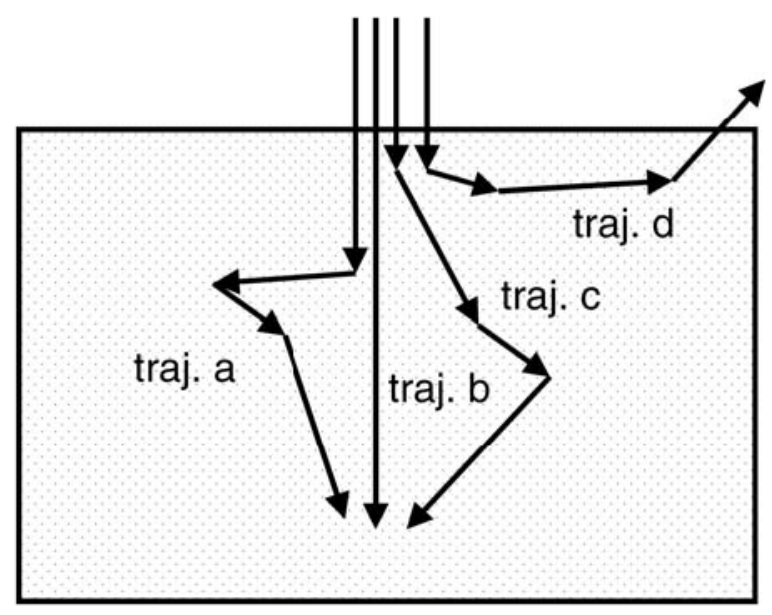

Figure 2.

A cartoon illustration of a simple photonic interpretation of scattering in a random medium. Photons, in relation to a given sample depth, can be categorized into three groups. The first group comprises ballistically propagating photons that do not experience any scattering during the propagation process; Trajectory $b$ is representative of the trajectories of such photons. Trajectories a and c are representatives of trajectories of photons that are scattered multiple times but which will still reach the given depth of interest. The last group of photons experience scattering in the medium and never make it to the depth of interest; Trajectory d is representative of the trajectories of such photons. 


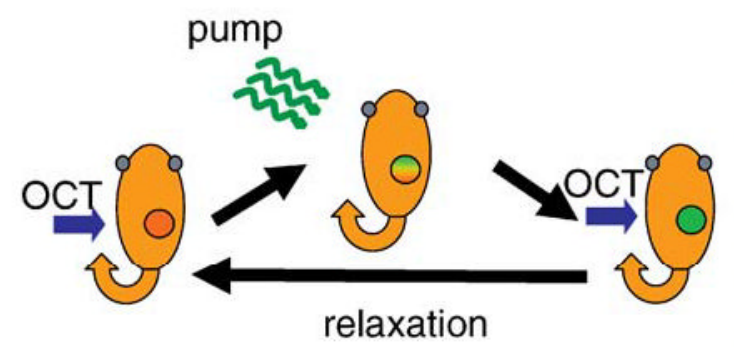

(a)

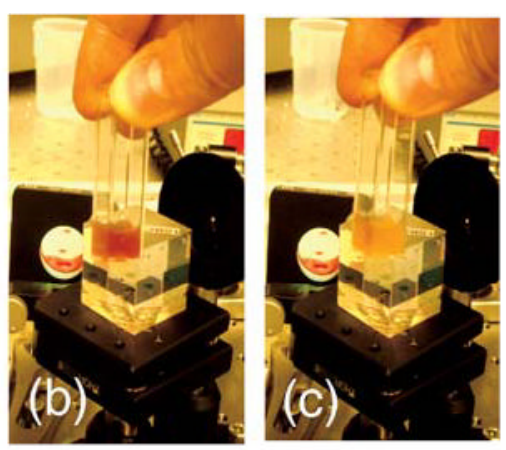

Figure 3.

(a) A cartoon illustration of optically excitable transition MCOCT scheme. OCT images acquired before and after a pump excitation that changed the absorption spectrum of the contrast agent can be processed to reveal the contrast agent's distribution. (b) and (c) show the absorption spectrum change induced in bactriorhodopsin by a $630 \mathrm{~nm}$ pump excitation. 

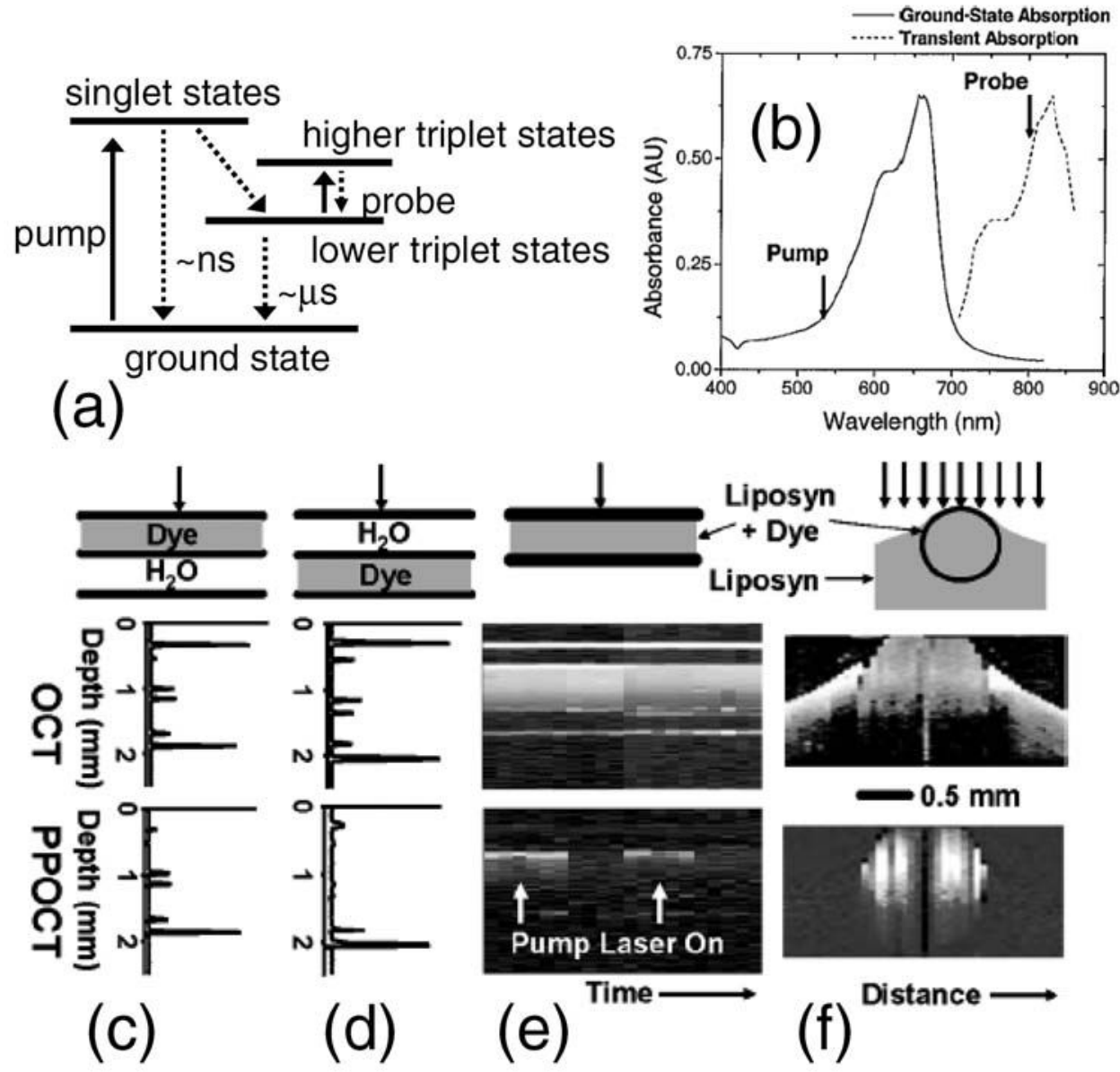

Figure 4.

(a) Energy level scheme of methylene blue. (b) The absorption spectrum of the ground state and the lower triplet state. The wavelengths of the pump and probe beams are indicated as well. (c) and (d) Linear plots of 50 averaged OCT and PPOCT A-scans in a two-level well phantom containing water and $500 \mu M$ methylene blue dye in water in alternate locations. Differential PPOCT signals appear at phantom interfaces below the level of the dye. (e) Log M-scans of OCT and PPOCT in a scattering medium ( $500 \mu M$ methylene blue dye in $0.5 \%$ Liposyn) with the pump laser alternatively blocked and unblocked. (f) Log OCT and PPOCT cross-sectional images of a capillary tube containing $500 \mu M$ methylene blue dye in $0.5 \%$ Liposyn, partially immersed in $0.5 \%$ Liposyn without dye. 

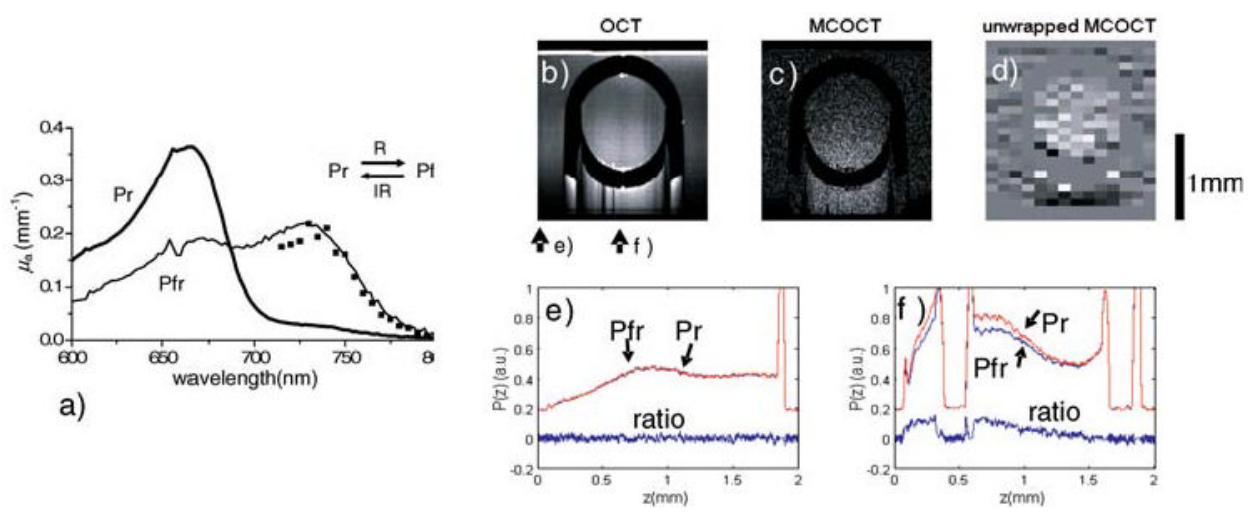

a)

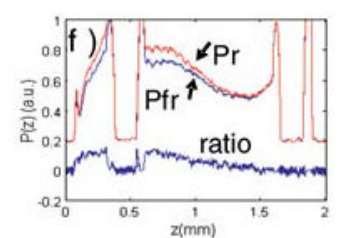

Figure 5.

(a) Absorption spectra of the two states of PhyA. (b) The $750 \mathrm{~nm}$ OCT B-scan with PhyA in Pr state (1.5 mm wide $\times 2 \mathrm{~mm}$ deep); the OCT B-scan with PhyA in Pfr state appears very similar (not shown). (c) MCOCT differential scan derived based on the operations described in Eq. (8). (d) Unwrapped MCOCT scan derived based on the operations described in Eq. (9). (e) A-scans with PhyA in Pr and Pfr state extracted from the locations indicated by the arrows in b. (f) A-scans with PhyA in Pr and Pfr state extracted from the locations indicated in b. 


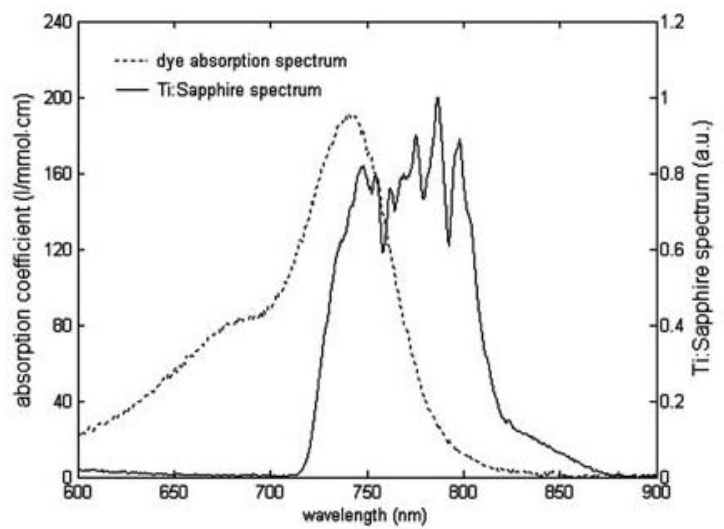

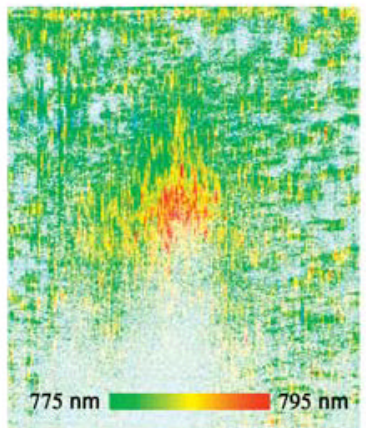

(a)

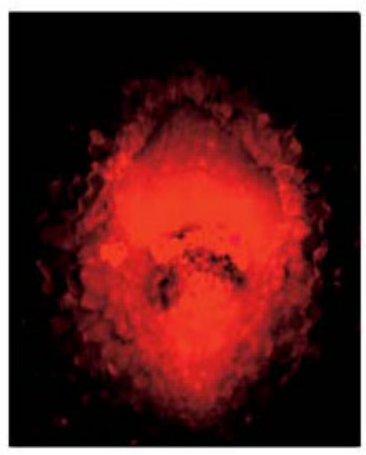

(c)

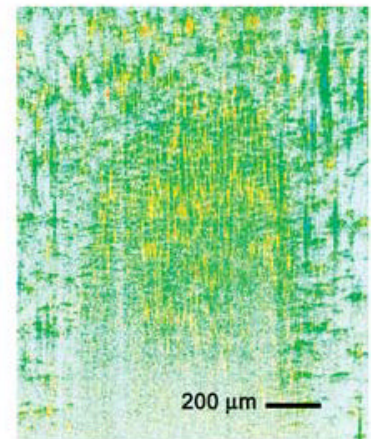

(b)

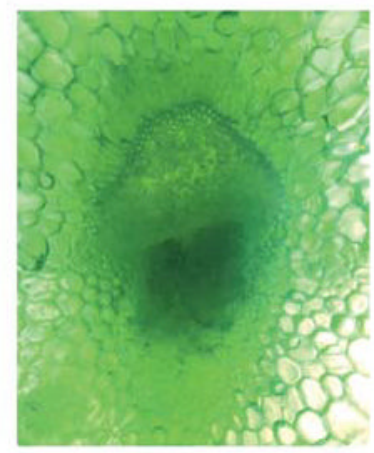

(d)

Figure 6.

Spectrum of the dye's absorption and the OCT probe laser used. (a) Spectroscopic OCT image of a celery stalk with dye present within the vascular bundle. The color bar shows the correspondence between pseudocolor labeling and the spectral centroid shift in the image. (b) Spectroscopic OCT image of the same area without dye. (c) Fluorescence microscopy and (d) light microscopy images showing the vascular bundle and the surrounding collenchyma tissue. 

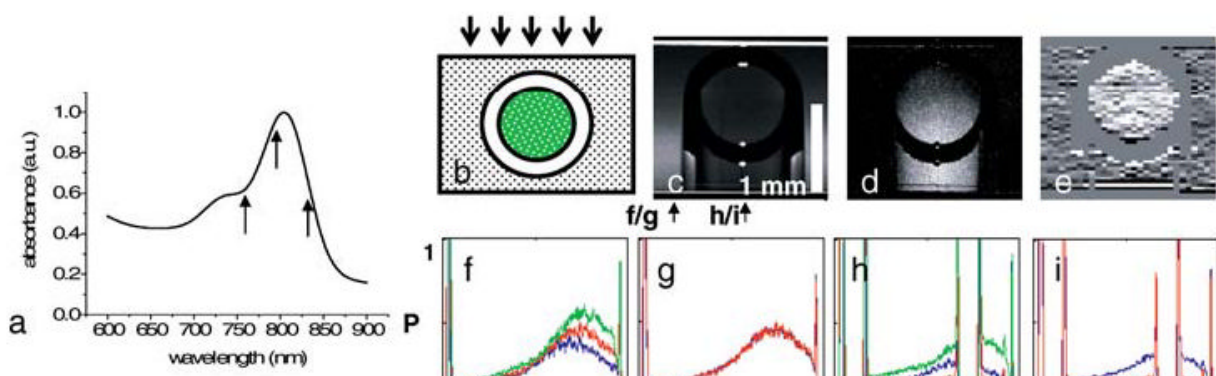

$\mathrm{f} / \mathrm{g} \uparrow \mathrm{h} / \mathrm{i}^{\dagger}$
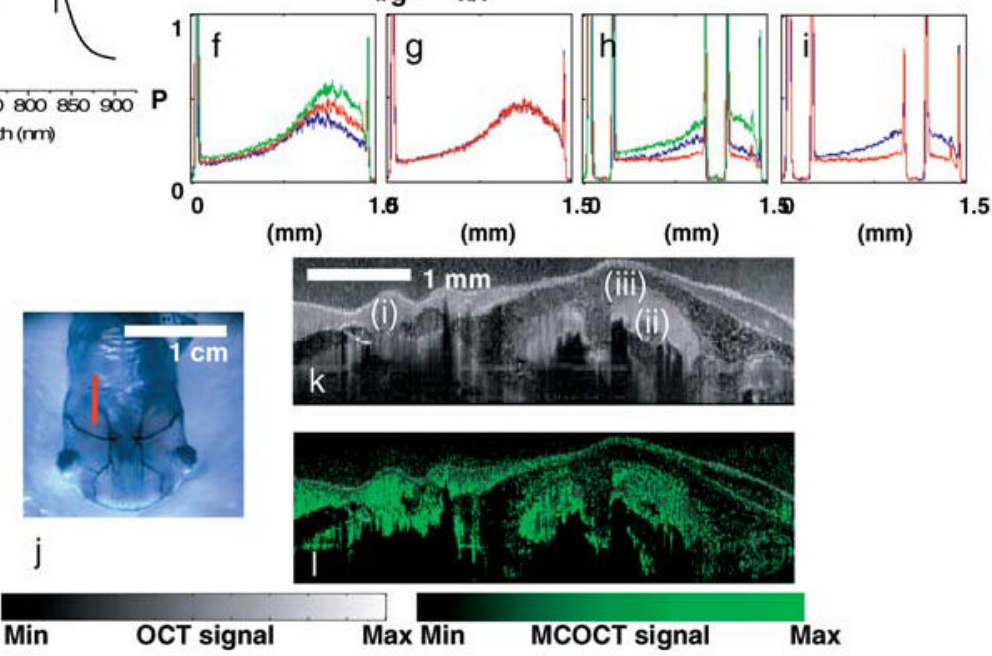

Figure 7.

(a) Absorption spectrum of ICG. (b) Cross-section of target sample; arrows indicate probe light direction, green represents location of ICG and scattering medium, and shade represents scattering medium. (c) OCT image at $795 \mathrm{~nm}$; arrows locate line traces from which Fig. 7(f)(i) are obtained. (d) Contrast image based on processing described in Eq. (12). (e) Unwrapped contrast image based on processing described in Eq. (9). (g) and (i) OCT A-scans at the different wavelengths (green, $760 \mathrm{~nm}$; red, $795 \mathrm{~nm}$; blue, $830 \mathrm{~nm}$ ). (h) and (j) Processed scans corresponding to the numerator (blue) and denominator (red) of the fraction within the logarithm in first line of Eq. (12). (j) Posterior view of a stage 54 X. laevis tadpole; red line indicates location where images are acquired. (k) OCT image; (i) parabranchial cavity, (ii) gill arches and (iii) opercular fold. (1) Composite image of the illuminated regions; the dye contrast is clearly visible within the gill arches and the parabranchial cavity. 


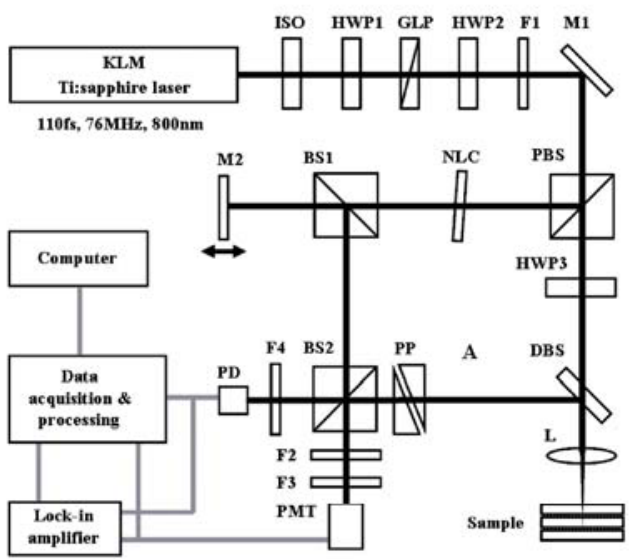

(a)
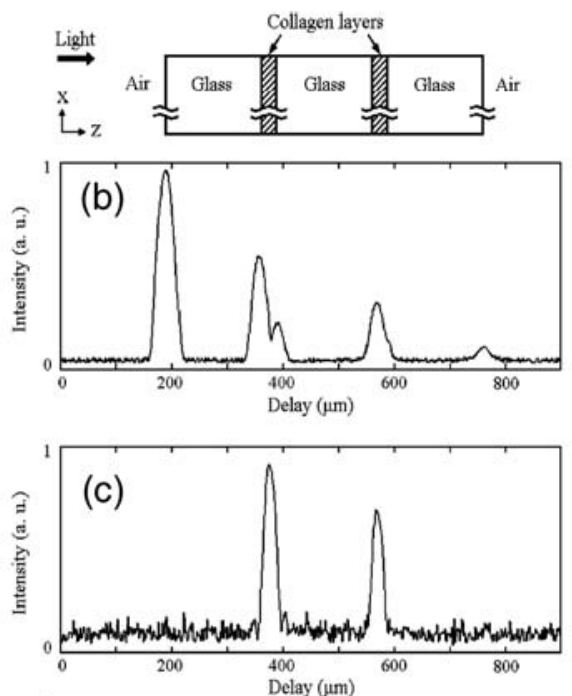

(d)

Figure 8.

(a) SH-OCT experimental setup: ISO, isolator; HWP1-HWP3, half-wave plates; GLP, Glan laser polarizer; F1-F4, filters; M1, M2, mirrors; PBS, polarization beam splitter; NLC, nonlinear b-barium borate crystal; BS1, BS2, broadband nonpolarization beam splitters; DBS, dichroic beam splitter; L, objective; PP, prism-pair dispersion compensator; PD, photodiode; PMT, photo-multiplier tube (from Ref. [71]). (b) and (c) SH-OCT and conventional OCT signals of one depth scan. The sample structure is shown at the top (from Ref. [74]). (d) Overlay of the SH-OCT image (green-red) onto the fundamental OCT image acquired simultaneously from a fish scale. The scale bar in the upper right corner is $250 \times 250 \mu \mathrm{m}$ (from Ref. [76]). 


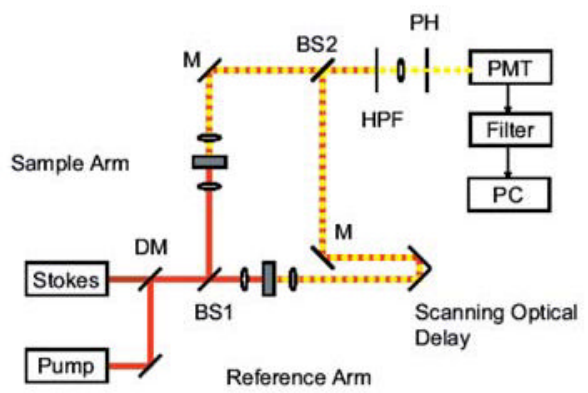

(a)

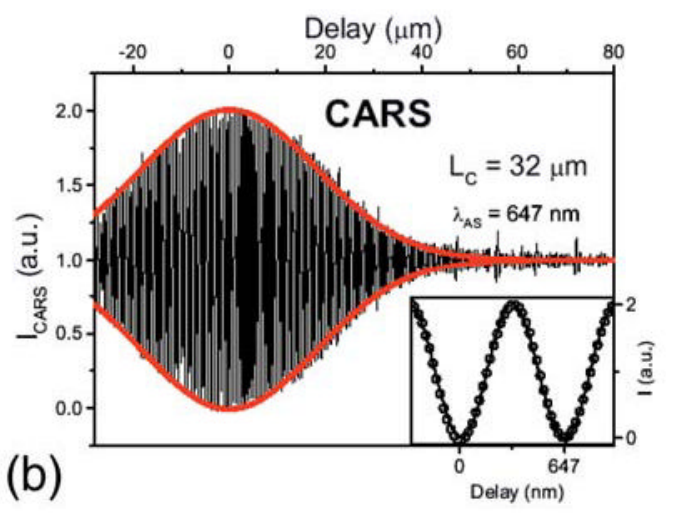

Figure 9.

(a) Setup of the interferometric CARS measurement system. DM, dichroic mirror; BS, beamsplitter; M, mirror; HPF, high pass filter; PH, pinhole; PMT photomultiplier tube; PC, personal computer. (b) CARS interferogram detected at the beamsplitter BS2 of the setup shown in (a). In the inset is shown a detail of the interference pattern and its fit by the real part of the degree of coherence function (open circles: experimental data; solid line: fit). $l_{c}$ is the coherence length of the pulse. $\lambda_{\mathrm{AS}}$ is the wavelength of the CARS signal. 


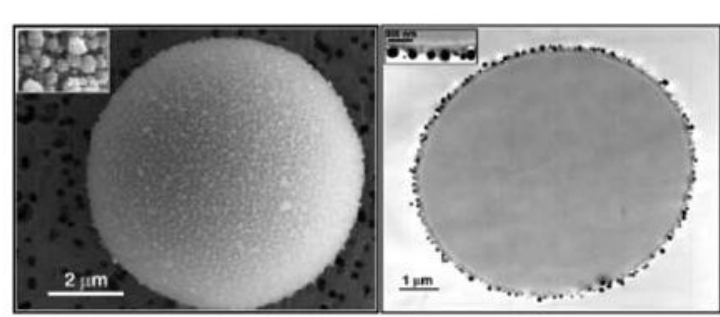

(a)

(b)

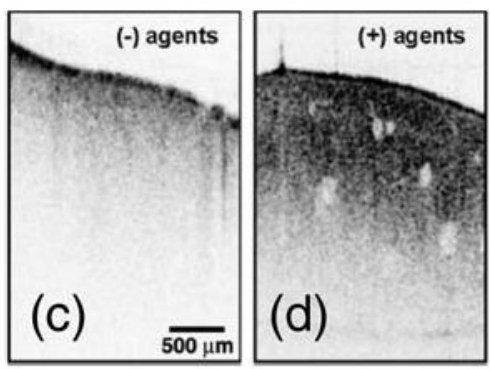

Figure 10.

Scanning- (a) and transmission- (b) electron micrographs of an oil-filled microsphere contrast agent, showing scattering silica nanoparticles in the shell. OCT images of mouse liver (c) without and (d) with gold-shelled, oil-filled microsphere contrast agents. 

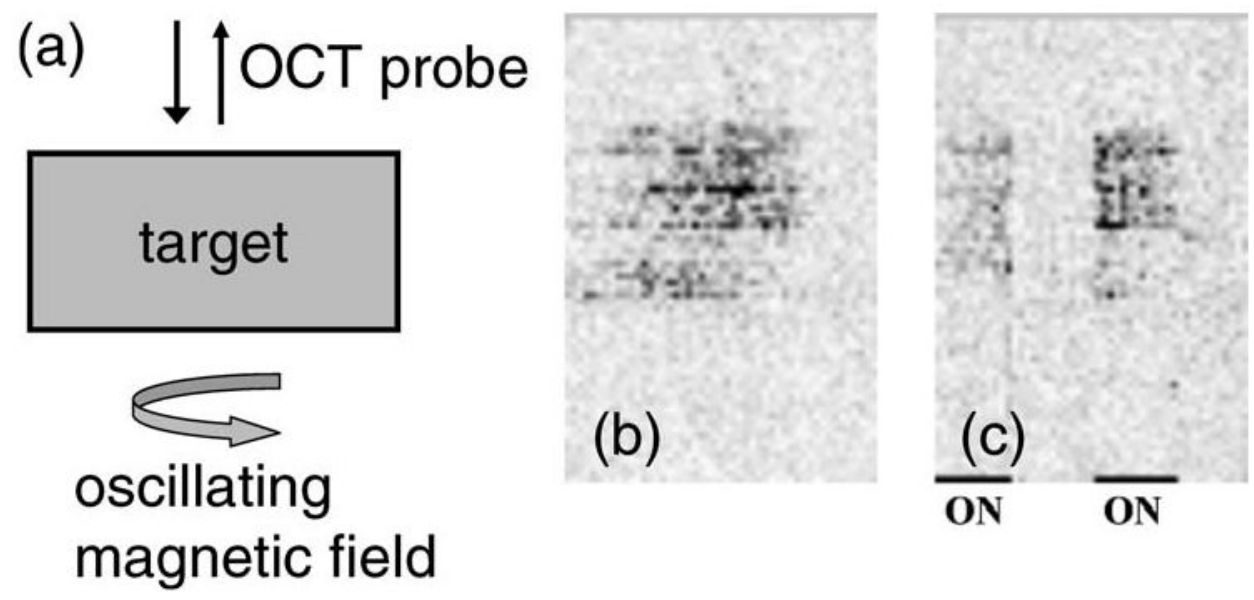

Figure 11.

(a) Cartoon illustration of the imaging scheme in magnetically modulated MCOCT. (b) Selective OCT imaging of macrophage cells (300 $\mathrm{mm}$ tall, $200 \mathrm{~mm}$ wide) using magnetic contrast. The image in (c) was acquired with the solenoid power supply on only for portions of the image indicated. This clearly indicates improved contrast in the presence of the magnetic field. 


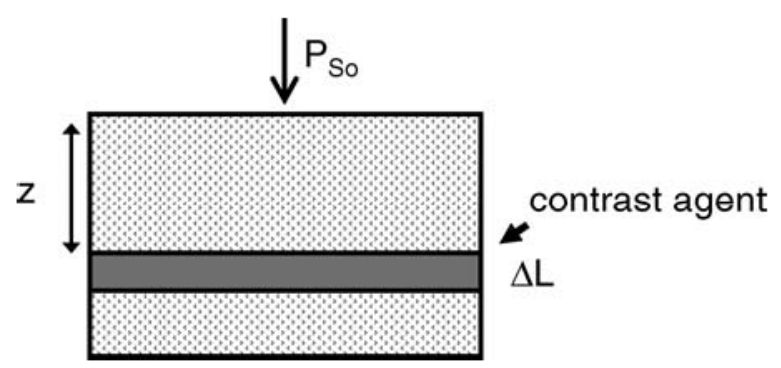

Figure 12.

Diagram of the proposed test sample for determining the various MCOCT sensitivity to contrast agents. 
Table 1

Summary of the parameters associated with the various reported MCOCT methods

\begin{tabular}{|c|c|c|c|c|c|c|}
\hline Method & $\begin{array}{c}\text { Contrast agent } \\
\text { concentration }(\mu M)\end{array}$ & Pulse duration (s) & $\begin{array}{c}\text { Max. } \\
\text { instantaneous } \\
\text { intensity (W/ } \\
\left.\mathbf{c m}^{2}\right)\end{array}$ & $\begin{array}{c}\text { Photodamage } \\
\text { threshold (W/ } \\
\left.\mathbf{c m}^{2}\right)\end{array}$ & $\begin{array}{c}\text { ANSI } \\
\text { (per } \\
\text { pulse) } \\
(\mathbf{W} / \\
\left.\mathbf{c m}^{2}\right)\end{array}$ & $\begin{array}{l}\text { Average } \\
\text { intensity } \\
\left(W / \mathrm{cm}^{2}\right)\end{array}$ \\
\hline Pump-probe & 500 & $2 \times 10^{-6}$ & $4 \times 10^{5}$ & $2 \times 10^{8}$ & $\begin{array}{l}8 \times \\
10^{2}\end{array}$ & $8 \times 10^{2}$ \\
\hline Pump suppression & 83 & $\mathrm{CW}$ & $1 \times 10^{3}$ & - & - & $1 \times 10^{3}$ \\
\hline Spectral triangulation & 200 & $\mathrm{CW}$ & 400 & - & - & 400 \\
\hline SH-OCT & Agent only & $1 \times 10^{-13}$ & $3 \times 10^{9}$ & $5 \times 10^{11}$ & - & $3 \times 10^{4}$ \\
\hline NIVI & Agent only & $7 \times 10^{-14}$ & $5 \times 10^{11}(\mathrm{est})$ & $4 \times 10^{11}$ & - & $9 \times 10^{3}$ \\
\hline Engineered microsphere & Microscopic particles & $\mathrm{CW}$ & $8 \times 10^{3}$ & - & - & $8 \times 10^{3}$ \\
\hline Magnetic particle & Microscopic particles & $\mathrm{CW}$ & $1 \times 10^{3}$ (est) & - & - & $\begin{array}{c}1 \times 10^{3} \\
(\mathrm{est})\end{array}$ \\
\hline
\end{tabular}

CW: continuous wave 